\title{
Nonhydrostatic Icosahedral Atmospheric Model (NICAM) for global cloud resolving simulations
}

\author{
M. Satoh ${ }^{\mathrm{a}, \mathrm{b}, *}$, T. Matsuno ${ }^{\mathrm{a}}, \mathrm{H}$. Tomita $^{\mathrm{a}}, \mathrm{H}$. Miura $^{\mathrm{a}}$, \\ T. Nasuno ${ }^{a}$, and S. Iga ${ }^{a}$ \\ ${ }^{a}$ Frontier Research Center for Global Change/ Japan Agency for Marine-Earth \\ Science and Technology, 3173-25 Showa-machi,Kanazawa-ku, Yokohama-shi, \\ Kanagawa,236-0001,Japan \\ ${ }^{\mathrm{b}}$ Center for Climate System Research, University of Tokyo, 5-1-5 Kashiwanoha, \\ Kashiwa-shi, Chiba, 277-8568, Japan
}

$\overline{0} 30$ June 2006, Submitted to J. Comp. Phys.; 15 January 2007; 1 February 2007, revised.

Preprint submitted to Elsevier Science

1 February 2007

(C) 2007. This manuscript version is made available under the Elsevier user license http://www.elsevier.com/open-access/userlicense/1.0/ 


\section{Abstract}

A new type of ultra-high resolution atmospheric global circulation model is developed. The new model is designed to perform "cloud resolving simulations" by directly calculating deep convection and meso-scale circulations, which play key roles not only in the tropical circulations but in the global circulations of the atmosphere. Since cores of deep convection have a few $\mathrm{km}$ in horizontal size, they have not directly been resolved by existing atmospheric general circulation models (AGCMs). In order to drastically enhance horizontal resolution, a new framework of a global atmospheric model is required; we adopted nonhydrostatic governing equations and icosahedral grids to the new model, and call it Nonhydrostatic ICosahedral Atmospheric Model (NICAM). In this article, we review governing equations and numerical techniques employed, and present the results from the unique $3.5 \mathrm{~km}-\mathrm{mesh}$ global experiments - with $O\left(10^{9}\right)$ computational nodes - using realistic topography and land/ocean surface thermal forcing. The results show realistic behaviors of multi-scale convective systems in the tropics, which have not been captured by AGCMs. We also argue future perspective of the roles of the new model in the next generation atmospheric sciences.

Key words: nonhydrostatic model, cloud resolving model, cloud clusters, icosahedral grids, atmospheric general circulation models, aqua-planet experiments

\footnotetext{
* Corresponding author : Masaki Satoh

Email address: satoh@ccsr.u-tokyo.ac.jp (M. Satoh).
} 


\section{Introduction}

Higher resolution computations are continuously demanded for global atmospheric modeling. Although the resolvable scale of global atmospheric models has been the order of $100 \mathrm{~km}$ until a few years ago, numerical simulations with resolvable scale of $O(10 \mathrm{~km})$ can be achieved due to recent massive computer facilities [1-3]. As the resolvable scale is coming down to less than $10 \mathrm{~km}$, however, traditional atmospheric general circulation models (AGCMs) encounter fundamental difficulties in the dynamic framework formulation as well as in the computational efficiency. As the resolution increases, AGCMs start capturing flow features with the scales of motion comparable in the horizontal and the vertical (e.g., deep convection, fine scale gravity waves), whereupon the hydrostatic approximation becomes invalid. Although deep convective systems in the tropics play key roles in the global atmospheric circulations, they have not been directly resolved by AGCMs, and their effects have been considered in parameterized form [4]. Effects of fine scale gravity waves that are parameterized as gravity wave drag in AGCMs become captured as well as the resolution increases, and propagation of such gravity waves is incorrectly calculated unless the nonhydrostatic effect is taken into account.

For the numerical algorithm, many of the existing AGCMs employ the spectral transform method to represent the spherical fields. It is pointed out, however, that as the horizontal resolution increases, the spectral transforms become inefficient for high performance computing; e.g., [5-9]. One of the serious problems is the computational inefficiency of the Legendre transformation: the computational operations are $O\left(N^{3}\right)$ complex, where $N$ is the truncation number. Another problem occurs for the computation on a massively parallel 
computer: the spectral transform method requires extensive data movement between computer nodes. Although the double Fourier transformation method is proposed by [9-12] as an alternative, this method still requires global communication between computer nodes.

To drastically increase the horizontal resolution, both of governing equations and numerical algorithms must be reconsidered for a new type of global atmospheric models. First, the nonhydrostatic dynamical cores must be chosen as the governing equations. Next, instead of the spectral methods, the grid point method is a prospective alternative for high-resolution modeling. As for the familiar latitude-longitude grid, however, the grid spacing near the poles becomes unaffordably small as the horizontal resolution is increased, so that numerical filters or reduced grids are required to avoid the computational instability near the poles, in general. In principle, the semi-Lagrangian, semiimplicit (SLSI) approach (cf. articles by Laprise, and by Staniforth and Wood in the same issue) might be employed to overcome severe restrictions of the time interval for the Courant-Friedrich-Lewy (CFL) condition. Several authors [13-19] have used the latitude-longitude grid to solve the set of nonhydrostatic equations with the SLSI approach to acquire a large time interval for integration. However, it is unclear how effective the elliptic solvers, developed for the SLSI schemes, would be for the stiff boundary value problems accompanying the ultra-high resolution calculations.

One can overcome the pole problem using other types of grid system with quasi-homogeneous grids over the sphere, such as icosahedral grids (references shown below), cubic grids [20-23], overset grids [24], and others [25]. As an example of the overset grids, [24] extended a nonhydrostatic model, MM5 (the PSU/NCAR mesoscale model [26]) to cover the global domain using two set 
of polar stereo coordinates. For the time integration scheme, the split-explicit scheme is used for horizontal propagation of fast waves with implicit treatment for vertical propagation [27], so that the use of the multi-dimensional elliptic solver is circumvented. However, their approach requires interpolating dependent variables around the overlapping regions so that conservation of physical quantities is not guaranteed. Although the use of overset grids is an easiest way to extend existing nonhydrostatic models to the global domain, physical properties derived from special treatment of the overlapping regions are unknown a priori.

The icosahedral grid, proposed originally by [28] and [29], is nowadays one of the major grid systems for atmospheric modeling. After early attempts of icosahedral grid modeling [30-34], the icosahedral models have been revisited in the recent context of high resolution modeling. Primitive (hydrostatic) equation global models using the icosahedral grid are being developed at Colorado State University for climate modeling (CSU AGCM) [35-37], Deutscher Wetterdienst for numerical prediction modeling (GME) [38, 39], and the Max Planck Institute for Meteorology (ICON) [40]. Another streams of icosahedral grid modeling for shallow water are found in [5, 41] and [42].

At Frontier Research Center for Global Change/ Japan Agency for MarineEarth Science and Technology (FRCGC/JAMSTEC), we are taking a unique approach to develop an icosahedral atmospheric model using the nonhydrostatic system. The new model is called NICAM (Nonhydrostatic ICosahedral Atmospheric Model). In preliminary studies, we have examined properties of icosahedral grids and improved numerical accuracy of the differential operators and homogeneity of the grid system using the spring dynamics $[43,44]$. Several techniques are proposed to achieve smoother icosahedral grids $[36,45]$; 
the spring dynamics modification by $[43,44]$ is one of the major approaches to construct the icosahedral grids.

NICAM is intended to be used for "cloud resolving simulations" by decreasing grid intervals below $5 \mathrm{~km}$ using the outstanding computing power of the Earth Simulator [46]. We have developed NICAM as a unified model in the sense that it can be used for both short term numerical predictions for weather systems such as a week, and long term simulations to obtain quasi-equilibrium climate states. (Such a unified model approach is also taken by [14, 19]. ) There are different types of nonhydrostatic global models [47]; incompressible, anelastic, and elastic systems can be considered. we use the fully compressible (elastic) nonhydrostatic system to obtain thermodynamically quasi-equilibrium states by long time simulations. For this purpose, we devised a nonhydrostatic numerical scheme which guarantees conservation of mass and energy [48, 49]. [50] implemented this nonhydrostatic scheme to the global model using the icosahedral grid configuration, and developed a dynamical core of NICAM. In [50], formulation and numerical scheme of the dynamical core of NICAM are described, together with numerical results of several test cases. [51] also showed numerical results of dynamical core experiments of extra-tropical cyclones in the context of consistency of horizontal and vertical grid ratios.

In essence, the numerical scheme of NICAM is an extension of that of the regional model $[48,49]$ to the global model using the modified icosahedral grid $[43,44]$. Details of the formulation of the dynamical core of NICAM is presented in [50]. The split-explicit time integration scheme [27] is used for horizontal propagation of waves, and implicit scheme is used for vertical propagation of sound/gravity waves; thus, multi-dimensional elliptic solvers are not required. To extend the original nonhydrostatic scheme to the global 
domain, we need to reformulate the set of equations shown in $[48,49]$ for the spherical geometry and modify the scheme suitable for the icosahedral grid configuration. The finite volume method is used for numerical discretization, so that total mass and energy over the domain is conserved; thus this model is suitable for long term climate simulation.

In this paper, we review the formulation of NICAM, together with the results of global cloud resolving simulations. In particular, we describe the governing equations including moist processes used for NICAM, as an extension of the dynamical core [50]. The results of the global experiments with $3.5 \mathrm{~km}-$ horizontal-mesh interval are reported by [52-54], and their computational performance is summarized by [8]. These are experiments under an aqua-planet condition [55], with full physics included (i.e., radiation, boundary layer, and cloud microphysics schemes). Since the formulation of the equations with moist process for NICAM has not been described yet, this paper documents the full physics model. In Section 2, governing equations of NICAM with moist processes are summarized; details of the formulation are supplemented in the appendices A and B. Numerical techniques of NICAM are described in Section 3. Representative results of global cloud resolving simulations are reviewed in Section 4, where the results from the aqua-planet experiment and a first result of a global cloud resolving simulation with realistic land and sea distributions are shown. In Section 5, after reviewing the background of development of NICAM, we will discuss scientific relevances and the future perspective of NICAM. We summarize the paper in Section 6 . 


\section{Governing Equations}

In this section, the governing equations with the full moist physics used for NICAM are summarized. The formulation of the "dry" dynamical core is described in [50]. For the moist formulation of NICAM, we follow that of the Cartesian coordinate model [49]. While the warm rain process without ice is described in [49], we extend this scheme to cover the spherical geometry and to take account of the effects of ice. We also introduce deep atmosphere dynamics as described in [50]. Here, we write the governing equations of NICAM in a compact operator form, as actually integrated in the model. Formal definitions of the operator notation are provided in Appendix A; whereas a derivation of the governing equations set, starting with more basic fluid equations, is outlined in Appendix B. In this section, the resultant set of the equations is summarized. Description of symbols is given in Table 1.

We emphasize two metric symbols: $\gamma \equiv r / r_{0}$ for deep atmosphere, and $G^{1 / 2} \equiv$ $(\partial z / \partial \xi)_{h}$, for the terrain-following coordinates, where the vertical coordinate $\xi$ is related to height $z$ as a monotonic function of $\xi=\xi(z)$ (Appendix A). Multiplying the original set of physical variables by $G^{1 / 2} \gamma^{2}$, we define following variables:

$$
\left(R, P, \mathbf{V}_{h}, W, E_{a}, Q_{n}\right)=G^{1 / 2} \gamma^{2}\left(\rho^{\prime}, p^{\prime}, \rho \mathbf{v}_{h}, \rho w, \rho e_{a}, \rho q_{n}\right)
$$

where $\rho^{\prime}$ and $p^{\prime}$ are perturbation fields of density and pressure from a basic state that satisfies the hydrostatic balance equation: $\rho^{\prime}=\rho-\rho_{s}$ and $p^{\prime}=p-p_{s}$ with

$$
0=-\frac{\partial p_{s}}{\partial z}-\rho_{s} g
$$


where $p_{s}$ and $\rho_{s}$ stand for pressure and density of a basic state, which depends only on height $z$. Note $\rho$ is the total density of moist air including contribution of water substances. $\mathbf{v}_{h}$ and $w$ are the horizontal velocity vector and the vertical velocity component, respectively; $e_{a}$ is the sensible heat part of internal energy defined by (B.14); $q_{n}$ stands for the $n$-th component of water substance: water vapor for $n=1$, liquid water for $2 \leq n \leq j_{\max }+1$, and solid water for $j_{\max }+2 \leq n \leq j_{\max }+k_{\max }+1$. The number of liquid water categories is $j_{\max }$ and that of solid water (ice) categories is $k_{\max }$ (see Appendix B).

Multiplying (B.6), (B.8), (B.9), (B.15), and (B.1)-(B.4) by factor $G^{1 / 2} \gamma^{2}$, and using (A.3) and (A.6)-(A.8), we obtain the following prognostic equations of deep atmosphere in the terrain-following coordinates:

$$
\begin{aligned}
& \frac{\partial R}{\partial t}+\tilde{\nabla}_{h 0} \cdot\left(\frac{\mathbf{V}_{h}}{\gamma}\right)+\frac{\partial}{\partial \xi}\left(\frac{\mathbf{V}_{h}}{\gamma} \cdot \mathbf{G}^{\mathbf{z}}+\frac{W}{G^{1 / 2}}\right)=G_{R}, \\
& \frac{\partial \mathbf{V}_{h}}{\partial t}+\tilde{\nabla}_{h 0} \frac{P}{\gamma}+\frac{\partial}{\partial \xi}\left(\mathbf{G}^{\mathbf{z}} \frac{P}{\gamma}\right)=G_{\mathbf{V}_{h}}, \\
& \frac{\partial W}{\partial t}+\gamma^{2} \frac{\partial}{\partial \xi}\left(\frac{P}{G^{1 / 2} \gamma^{2}}\right)+R g=G_{W} \\
& \frac{\partial E_{a}}{\partial t}+\tilde{\nabla}_{h 0} \cdot\left(h_{a} \frac{\mathbf{V}_{h}}{\gamma}\right)+\frac{\partial}{\partial \xi}\left[h\left(\frac{\mathbf{V}_{h}}{\gamma} \cdot \mathbf{G}^{\mathbf{z}}+\frac{W}{G^{1 / 2}}\right)\right] \\
& -\left\{\mathbf{v}_{h} \cdot\left[\tilde{\nabla}_{h 0} \frac{P}{\gamma}+\frac{\partial}{\partial \xi}\left(\mathbf{G}^{\mathbf{z}} \frac{P}{\gamma}\right)\right]+w\left[\gamma^{2} \frac{\partial}{\partial \xi}\left(\frac{P}{G^{1 / 2} \gamma^{2}}\right)+R g\right]\right\}+W g \\
& =G_{E_{a}}, \quad \\
& \frac{\partial Q_{n}}{\partial t}+\tilde{\nabla}_{h 0} \cdot\left(q_{n} \frac{\mathbf{V}_{h}}{\gamma}\right)+\frac{\partial}{\partial \xi}\left[q_{n}\left(\frac{\mathbf{V}_{h}}{\gamma} \cdot \mathbf{G}^{\mathbf{z}}+\frac{W}{G^{1 / 2}}\right)\right]=G_{Q_{n}},
\end{aligned}
$$

where $\mathbf{G}^{\mathbf{z}} \equiv \nabla_{h 0} \xi$ which is defined in Appendix A. In (1)-(4), the left hand side terms are associated with fast propagating waves, while the right hand side terms are related to slow motions. This categorization is used for the time integration scheme in Section 3.3. The terms on the right hand sides are expressed as 


$$
\begin{aligned}
G_{R}= & -\sum_{n} \frac{\partial}{\partial \xi}\left(\frac{W_{n}^{*}}{G^{1 / 2}}\right) \\
G_{\mathbf{V}_{h}}= & \tilde{\mathbf{F}}_{h}-\tilde{\mathbf{A}}_{h}-\tilde{\mathbf{C}}_{h}-\sum_{n} \frac{\partial}{\partial \xi}\left(\mathbf{v}_{h} \frac{W_{n}^{*}}{G^{1 / 2}}\right) \\
G_{W}= & \tilde{F}_{z}-\tilde{A}_{z}-\tilde{C}_{z}-\sum_{n} \frac{\partial}{\partial \xi}\left(w \frac{W_{n}^{*}}{G^{1 / 2}}\right) \\
G_{E_{a}}= & -\sum_{n} \frac{\partial}{\partial \xi}\left(C_{n} T \frac{W_{n}^{*}}{G^{1 / 2}}\right)-\sum_{n} W_{n}^{*} g \\
& -\left(\mathbf{v}_{h} \cdot \tilde{\mathbf{F}}_{h}+w \tilde{F}_{z}\right)-L_{v 00} \tilde{S}_{v}+L_{f 00} \tilde{S}_{i}+\tilde{Q}_{h e a t}, \\
G_{Q_{n}}= & \tilde{S}_{n}-\frac{\partial}{\partial \xi}\left(\frac{W_{n}^{*}}{G^{1 / 2}}\right) .
\end{aligned}
$$

where $W_{n}^{*}$ is the mass flux of precipitable water substances, defined by

$$
W_{n}^{*}=G^{1 / 2} \gamma^{2} \rho q_{n} w_{n}^{*}
$$

$w_{n}^{*}$ is the terminal velocity relative to the air, and is non-zero only for precipitable water substances: e.g., "rain" and "snow". $C_{n}$ is the specific heat of the $n$-th component of water substance: $C_{n}=C_{l}$ for liquid water and $C_{n}=C_{i}$ for ice. $\left(\tilde{\mathbf{F}}, \tilde{Q}_{h e a t}, \tilde{S}_{*}\right) \equiv G^{1 / 2} \gamma^{2}\left(\mathbf{f}, q_{\text {heat }}, s_{*}\right)$ (See Appendix B for definitions). Vectors of the advection of momentum and the Coriolis force are $(\tilde{\mathbf{A}}, \tilde{\mathbf{C}})=G^{1 / 2} \gamma^{2}(\mathbf{a}, \mathbf{c})$, and $\tilde{\mathbf{A}}_{h}$ and $\tilde{A}_{z}$ are the horizontal and vertical components of $\tilde{\mathbf{A}}$. We introduce an orthogonal basis $\left\{\mathbf{e}_{1}, \mathbf{e}_{2}, \mathbf{e}_{3}\right\}$ fixed to the earth, where $\mathbf{e}_{3}$ is in the same direction as $\boldsymbol{\Omega}$, and define the three components of velocity by $\left(v_{1}, v_{2}, v_{3}\right)=\mathbf{v} \cdot\left(\mathbf{e}_{1}, \mathbf{e}_{2}, \mathbf{e}_{3}\right)$, as shown by Fig. 1 . In this case, $(\tilde{\mathbf{A}}, \tilde{\mathbf{C}})$ are specifically expressed as

$$
\begin{aligned}
\tilde{\mathbf{A}} & \equiv\left\{\tilde{\nabla}_{h 0} \cdot\left(v_{1} \frac{\mathbf{V}_{h}}{\gamma}\right)+\frac{\partial}{\partial \xi}\left[v_{1}\left(\frac{\mathbf{V}_{h}}{\gamma} \cdot \mathbf{G}^{\mathbf{z}}+\frac{W}{G^{1 / 2}}\right)\right]\right\} \mathbf{e}_{1} \\
& +\left\{\tilde{\nabla}_{h 0} \cdot\left(v_{2} \frac{\mathbf{V}_{h}}{\gamma}\right)+\frac{\partial}{\partial \xi}\left[v_{2}\left(\frac{\mathbf{V}_{h}}{\gamma} \cdot \mathbf{G}^{\mathbf{z}}+\frac{W}{G^{1 / 2}}\right)\right]\right\} \mathbf{e}_{2} \\
& +\left\{\tilde{\nabla}_{h 0} \cdot\left(v_{3} \frac{\mathbf{V}_{h}}{\gamma}\right)+\frac{\partial}{\partial \xi}\left[v_{3}\left(\frac{\mathbf{V}_{h}}{\gamma} \cdot \mathbf{G}^{\mathbf{z}}+\frac{W}{G^{1 / 2}}\right)\right]\right\} \mathbf{e}_{3} \\
\tilde{\mathbf{C}} & \equiv \rho G^{1 / 2} \gamma^{2}\left(-2|\Omega| v_{2} \mathbf{e}_{1}+2|\Omega| v_{1} \mathbf{e}_{2}\right) .
\end{aligned}
$$




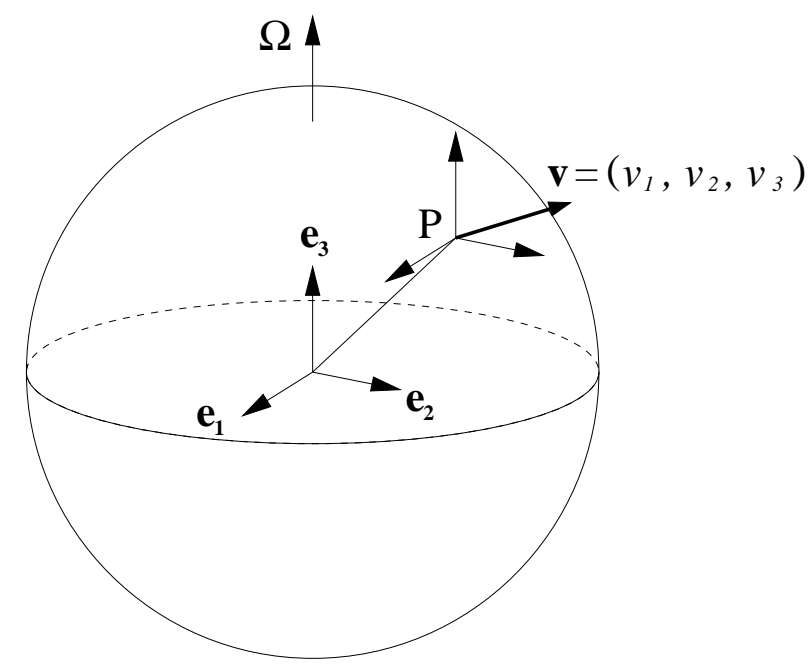

Fig. 1. Definitions of three components of velocity vector $\mathbf{v}$ and an orthogonal basis $\left\{\mathbf{e}_{1}, \mathbf{e}_{2}, \mathbf{e}_{3}\right\}$. An orthogonal basis is fixed to the earth with $\mathbf{e}_{3}$ parallel to the rotation vector $\boldsymbol{\Omega}$. The velocity vector $\mathbf{v}$ defined at point $\mathrm{P}$ is decomposed of three components $\left(v_{1}, v_{2}, v_{3}\right)$ in the directions of $\left\{\mathbf{e}_{1}, \mathbf{e}_{2}, \mathbf{e}_{3}\right\}$.

For numerical integration, we supplementary use the equation of the total energy, which can be given from (B.18):

$$
\begin{aligned}
& \frac{\partial\left(E_{a}+K+\Phi\right)}{\partial t}+\tilde{\nabla}_{h 0} \cdot\left[\left(h_{a}+k+\phi\right) \frac{\mathbf{V}_{h}}{\gamma}\right] \\
& +\frac{\partial}{\partial \xi}\left[\left(h_{a}+k+\phi\right)\left(\frac{\mathbf{V}_{h}}{\gamma} \cdot \mathbf{G}^{\mathbf{z}}+\frac{W}{G^{1 / 2}}\right)\right]=G_{E_{a}+K+\Phi}
\end{aligned}
$$

where $(K, \Phi)=\rho G^{1 / 2} \gamma^{2}(k, \phi)$, which are kinetic energy and potential energy respectively, and

$$
G_{E_{a}+K+\Phi}=-\sum_{n} \frac{\partial}{\partial \xi}\left(C_{n} T \frac{W_{n}^{*}}{G^{1 / 2}}\right)-L_{v 00} \tilde{S}_{v}+L_{f 00} \tilde{S}_{i}+\tilde{Q}_{h e a t}
$$




\section{Numerical methods}

\subsection{Overview of the numerical methods}

The governing prognostic equations solved in NICAM are (1)-(4) for the dy-

namical part, and (5) for water components. Eqs.(1)-(4) are equivalent to (28)-(31) of [50] except for the details of source terms. We use the same discretization method as [50] to integrate these equations. For the horizontal discretization, we use the icosahedral grid system on the sphere. Figure 2 exemplifies a series of consecutive icosahedral grids. The original icosahedron consists of 20 triangles; we called it "glevel-0" (grid division level 0). By dividing each triangles into four small triangles recursively, we obtain one-higher resolution with "glevel-n" (grid division level $n$ ). The total number of grid points is $N_{g}=10\left(2^{n}\right)^{2}+2$. We further modify the horizontal grid structure by spring dynamics to obtain smooth distribution of horizontal mesh [43, 44]; see Section 3.2. The Lorenz grid is used for the vertical grid configuration (Fig. 3). Horizontally, all the prognostic variables are defined at the same collocation points at the mass centers of control volumes (hexagon or pentagon). Vertically, $R, \mathbf{V}_{h}, E$ are defined at the integer levels and $W$ is defined at the half-integer levels as shown in Fig. 3. Water components $Q_{n}$ are defined at the same points as $E$.

There is an ongoing debate for the choice of the vertical grid system, i.e., the Lorenz grid vs the Charney-Phillips grid [56-59]. In the Lorenz grid, both thermodynamic variables are staggered with respect to vertical velocity, while temperature and vertical velocity are defined at the same vertical levels in the Charney-Phillips grid. So far, we have not encountered any difficulties with 
(a)

(b)
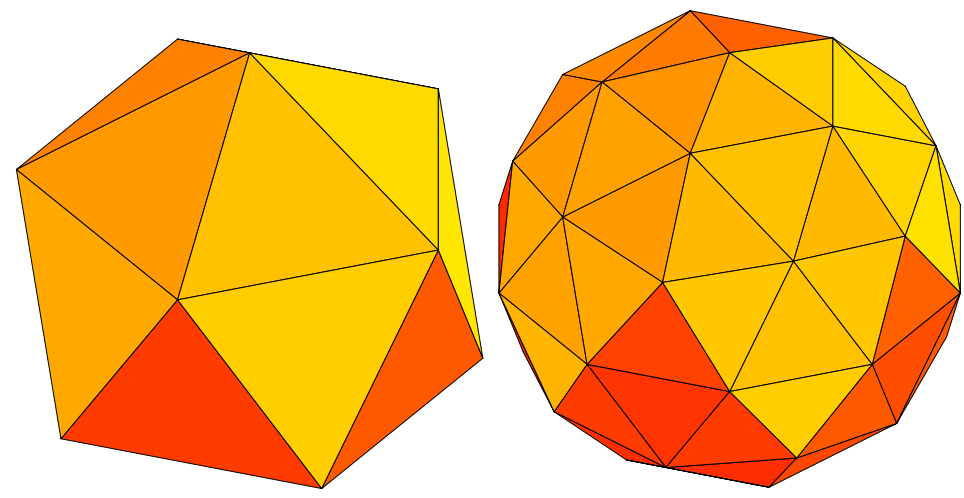

(c)
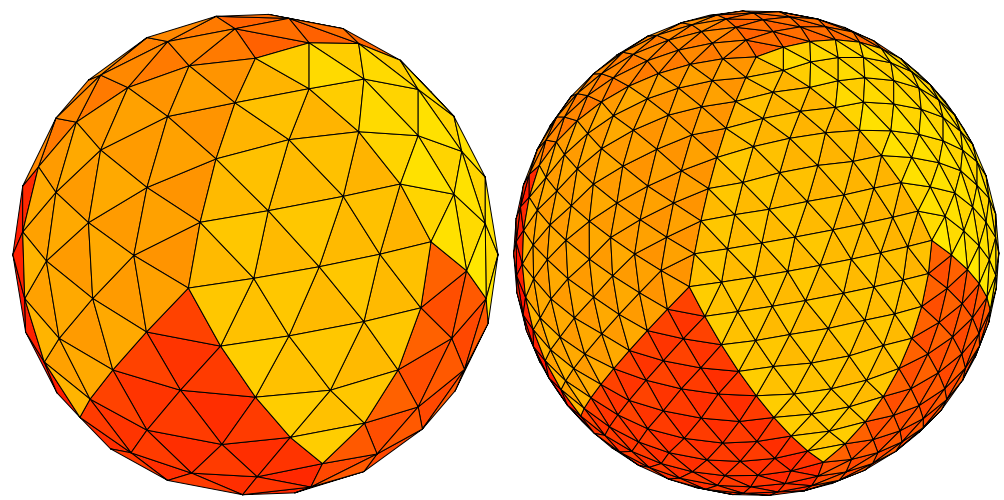

(d)

Fig. 2. The icosahedral grids with glevel-0 (a), 1 (b), 2 (c), and 3 (d).

the Lorenz grid. We believe that the Lorenz grid is appropriate for the nonhydrostatic system, where the three-dimensional divergence is defined at the same point as thermodynamic variables and deviations from the hydrostatic balance are automatically adjusted in the non-hydrostatic system. Nonetheless, we appreciate that properties of the numerical modes reported by [51] may depend on the vertical grid system.

We use the split-explicit scheme to integrate the governing equations. Figure 4 outlines the time integration procedure. The fast mode terms are evaluated at every small time step with a time interval $\Delta \tau$, while the slow mode terms are evaluated at large time steps with a larger time interval $\Delta t$, thus defining the split number $N_{s}=\Delta t / \Delta \tau$. For the small time step integration, we implicitly 


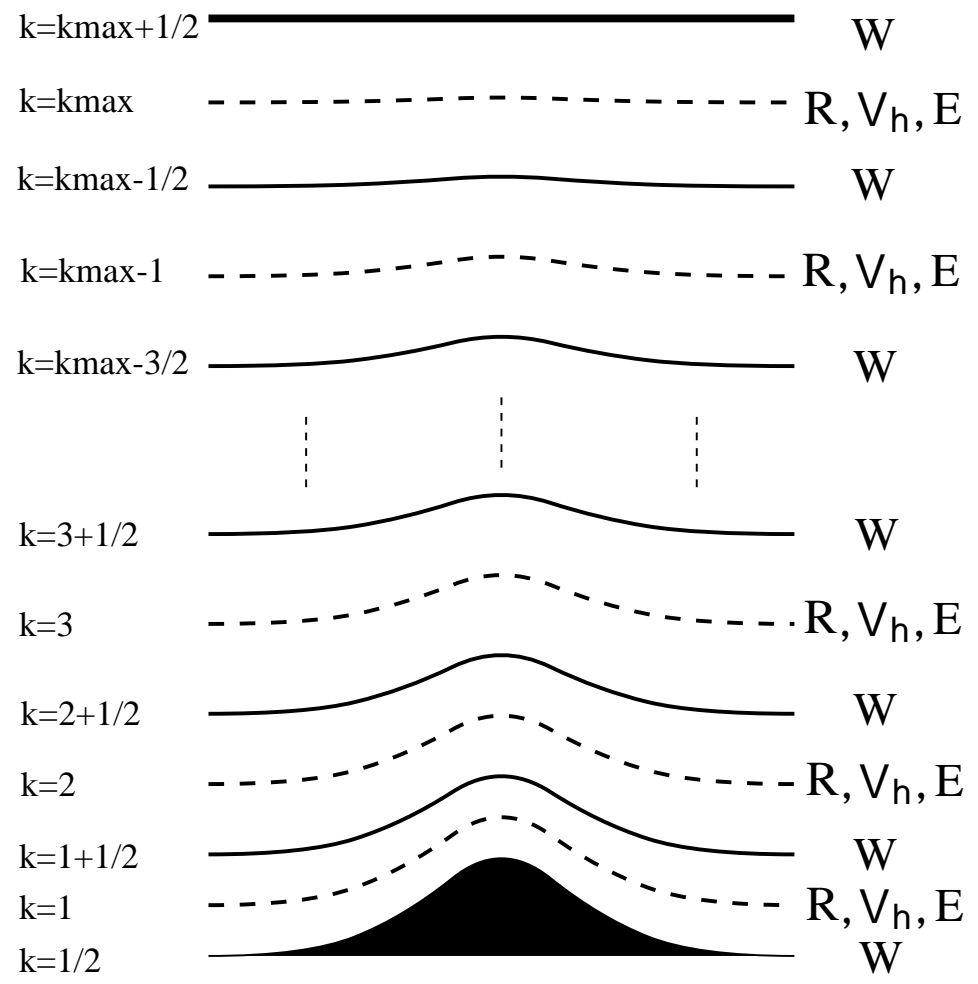

Fig. 3. Vertical levels and locations of variables of the Lorenz grids. $R$ is density, $V_{h}$ is horizontal velocity, $W$ is vertical velocity, and $E$ is internal energy. Water components $Q_{n}$ are defined at the same points as $E$. $k$ is the index of the vertical levels, and the number of the vertical levels is $k_{\max }$.

solve vertical propagation of fast waves and explicitly solve horizontal propagation of fast waves; this method is referred to as the horizontally explicit and vertically implicit scheme (HEVI; $[60,61])$. We use the forward-backward scheme based on the HEVI scheme and the flux division method [48], discussed later in section 3.3. To guarantee the conservation of total energy, the energy correction step is introduced using the equation of total energy (13) [48, 49]; see section 3.3 for further discussion.

For the large time step integration, we use the 2nd-order Runge-Kutta scheme (Fig. 4), and allow the 3rd-order Runge-Kutta scheme as an option for enhanced numerical stability $[62,63]$. The advection terms of momentum and 


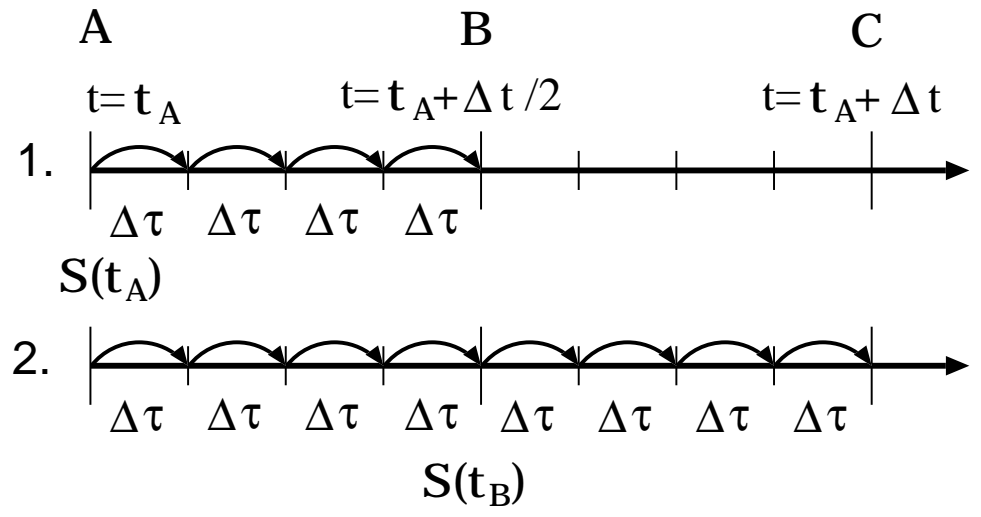

Fig. 4. Procedures of time integration. Case for the splitting number $N_{s}=8$ with the 2nd-order Runge-Kutta scheme. NICAM also introduces the 3rd-order Runge-Kutta scheme as an option.

physical processes are evaluated at large time steps.

\subsection{Icosahedral discretization}

We use the icosahedral grid for the horizontal mesh structure. For numerical modeling using the icosahedral grid, different approaches have been proposed for grid-point allocation, together with the choice of the prognostic variables [64]. [35, 36] employed the Z-grid [65] using potential vorticity and divergence as prognostic variables. Although it is a good choice to represent geostrophic adjustment, two-dimensional elliptic solvers are required for the time integration. [66] modified this scheme so as to guarantee conservation of total energy and potential enstrophy, and then the ZM-grid is developed by [67] using velocity components as prognostic variables to avoid use the elliptic solver. The ZM-grid is the staggering grid system in which velocity components and mass are defined at different points. Since the number of definition points of velocity is twice as many as those of mass, the ZM-grid has computational modes. In [67], several methods are proposed to filter out the computational modes of 
the ZM-grid.

$[5,39,41]$ use the velocity itself as the prognostic variable and allocate all the prognostic variables at the same triangle vertices; this grid system is equivalent to so-called the A-grid. We also employ the A-grid for our global nonhydrostatic model. The A-grid system does not contain computational modes, except for two-grid checker board noise. Besides, the A-grid has an advantage for data-handling and communication on the parallel computing. However, it is difficult to give physical meanings for the two-grid scale waves [65], so they should be regarded as a grid noise. The A-grid system has less horizontal resolution than the Z-grid or the ZM-grid if the same number of the grid points are used, and requires stronger numerical filters to remove grid noises.

We construct the icosahedral grid by a recursive division method [5]. Since areas of such obtained triangles are not smoothly distributed on the sphere, we need modification of grid arrangement to achieve second-order accuracy of descretization [43]. We modify the location of grid points by using spring dynamics as shown by Fig. 5 . In this process, the parameter $\beta$ defined in [43] is chosen as $\beta=1.15 ; \beta$ is associated with natural spring length, [44] shows that this value gives a well optimized grid structure from the viewpoints of both numerical accuracy and computational efficiency. After this modification. the control volume is defined by connecting the mass centers of triangular elements. The shape of control volume is hexagonal except for the pentagon at the twelve vertices of the original icosahedron. We furthermore relocate all the definition points of the prognostic variables to the mass centers of the control volumes. The icosahedral grid by the $n$-th time recursive division is referred to glevel- $n$. Figure 6 shows the modified icosahedral grid for glevel-5 with $\beta=1.15$. [43] showed that these modifications guarantee the second- 


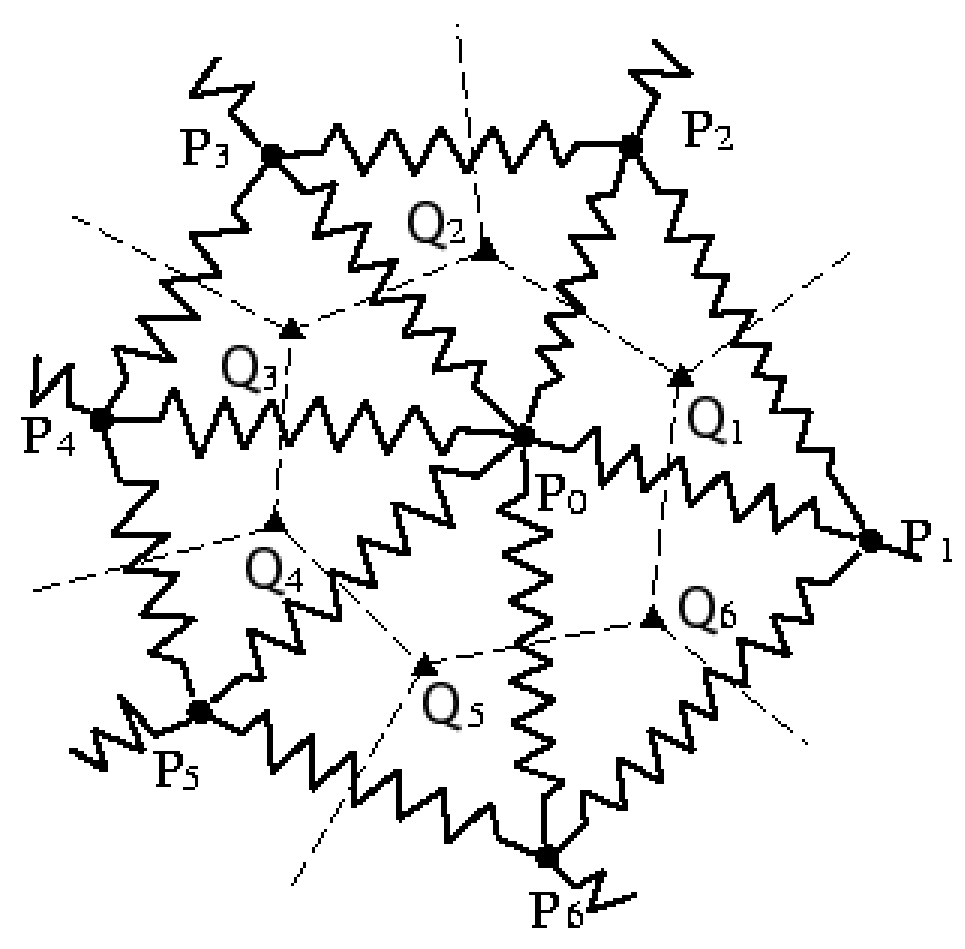

Fig. 5. Connection of springs for the modification of the grid points using spring dynamics.

order accuracy for horizontal differential operators at all the grid points when their discretization method is used. [45] compare various modification methods and examine properties of different icosahedral grids.

The arrangement of definition points and horizontal control volume is schematically shown by Fig. 7 . If a set of vectors $\mathbf{u}$ is given at all the vertices of triangles $P_{i}$ in Fig.7, vectors $\mathbf{u}$ at the vertices of control volume $Q_{i}$ are interpolated as

$$
\mathbf{u}_{h}\left(Q_{i}\right) \simeq \frac{s_{0} \mathbf{u}_{h}\left(P_{0}\right)+s_{1} \mathbf{u}_{h}\left(P_{i}\right)+s_{2} \mathbf{u}_{h}\left(P_{1+\bmod (i, 6)}\right)}{s_{0}+s_{1}+s_{2}}
$$

where $s_{0}, s_{1}$, and $s_{2}$ are the areas of $Q_{i} P_{i} P_{1+\bmod (i, 6)}, Q_{i} P_{1+\bmod (i, 6)} P_{0}$, and $Q_{i} P_{0} P_{i}$, respectively. The number 6 is replaced with 5 at the pentagonal control volumes. The divergence is calculated from the Gauss theorem as 


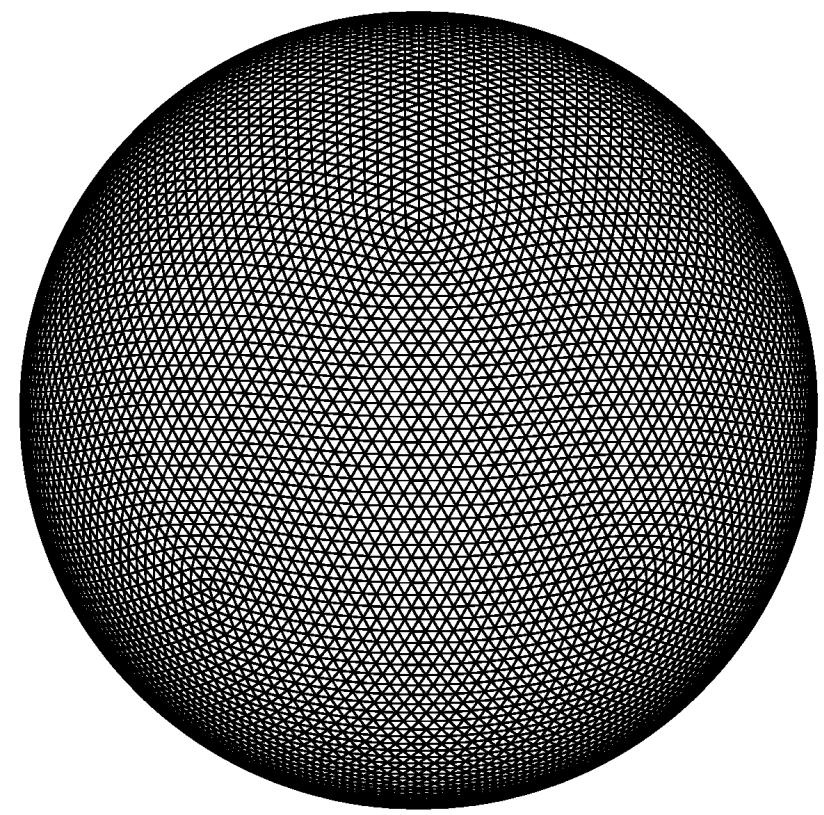

Fig. 6. Modified icosahedral grid by spring dynamics (glevel-5).

$$
\nabla_{h 0} \cdot \mathbf{u}_{h}\left(P_{0}\right) \simeq \frac{1}{a\left(P_{0}\right)} \sum_{i=1}^{6} b_{i} \frac{\mathbf{u}_{h}\left(Q_{i}\right)+\mathbf{u}_{h}\left(Q_{1+\bmod (i, 6)}\right)}{2} \cdot \mathbf{n}_{i},
$$

where $b_{i}$ and $\mathbf{n}_{i}$ denote the geodesic arc length of $Q_{i} Q_{1+\bmod (i, 6)}$ and the outward unit vector normal to this arc at the midpoint of $Q_{i} Q_{1+\bmod (i, 6)} \cdot a\left(P_{0}\right)$ is the area of control volume associated with the point $P_{0}$.

The gradient operator to an arbitrary variable $\phi$ is calculated as

$$
\nabla_{h 0} \phi\left(P_{0}\right) \simeq \frac{1}{a\left(P_{0}\right)} \sum_{i=1}^{6} b_{i} \frac{\phi\left(Q_{i}\right)+\phi\left(Q_{1+\bmod (i, 6)}\right)}{2} \mathbf{n}_{i}-\frac{\phi_{0}}{a\left(P_{0}\right)} \sum_{i=1}^{6} b_{i} \mathbf{n}_{i}
$$

where $\phi\left(Q_{i}\right)$ is interpolated by the similar way to (15). In general, the gradient vector given by (17) contains a vertical component, since the allocation points are on the sphere. We simply set the vertical component of the gradient vector equal to zero after the operation of (17).

[43] examined properties of differential operators and convergence of the shallow water solutions. Figure 8 shows resolution dependency of the error norms 
of divergence operator $\nabla \cdot \mathbf{u}$ where $\mathbf{u}=\sin \lambda \nabla\left(\cos 3 \lambda \cos ^{4} 3 \theta\right) ; \theta$ and $\lambda$ are latitude and longitude, respectively. The error norms are defined by

$$
l_{2}(x)=\frac{\left(I\left[\left(x-x_{t}\right)^{2}\right]\right)^{1 / 2}}{\left(I\left[x_{t}^{2}\right]\right)^{1 / 2}}, \quad l_{\infty}(x)=\frac{\max \left|x-x_{t}\right|}{\max \left|x_{t}\right|}
$$

$x_{t}$ is the exact solution and $I$ denotes the global-averaging operator. Compared to the standard grid (denoted by STD-grid), the modified grid (denoted by SPR-GC-grid) shows better convergence, particularly seen in the infinity norm $l_{\infty}$. Figure 9 is the error norm of height $h$ for the shallow water equations with rigid body rotation (Williamson's test case 2; [68]). "STD-grid" is the standard grid without any modification and "SPR-GC-grid" is the modified grid by spring dynamics; "GC" means further modification by moving the definition points of variables to the gravitation centers of control volumes; see [43]. The numerical solution with the modified grid (SPR-GC-grid) is stable for 5-days integration, while the solutions with the standard grids (STD-grid, STD-GC-grid) have much errors as times goes on. As resolutions become finer from glevel-4 to glevel-7, the infinity norms of the numerical solutions with the modified grid systematically decreases; the second-order accuracy is achieved by this method.

The evaluation of the advection terms of momentum would contain the metric terms if the spherical coordinates are used. In order to avoid explicit treatment of the metric terms, we use orthogonal basis $\left\{\mathbf{e}_{1}, \mathbf{e}_{2}, \mathbf{e}_{3}\right\}$ independent of positions for the evaluation of the advection terms. The velocity vector $\mathbf{V}$ is composed of $\mathbf{V}_{h}$ and $W$, whose governing equations are (2) and (3), respectively. For time integration of $\mathbf{V}_{h}$, we use three Cartesian components of horizontal velocity vector: $\left(V_{h 1}, V_{h 2}, V_{h 3}\right)=\mathbf{V}_{h} \cdot\left(\mathbf{e}_{1}, \mathbf{e}_{2}, \mathbf{e}_{3}\right)$. That is, the prognostic equation of the horizontal velocity vector (2) consists of three equations, 


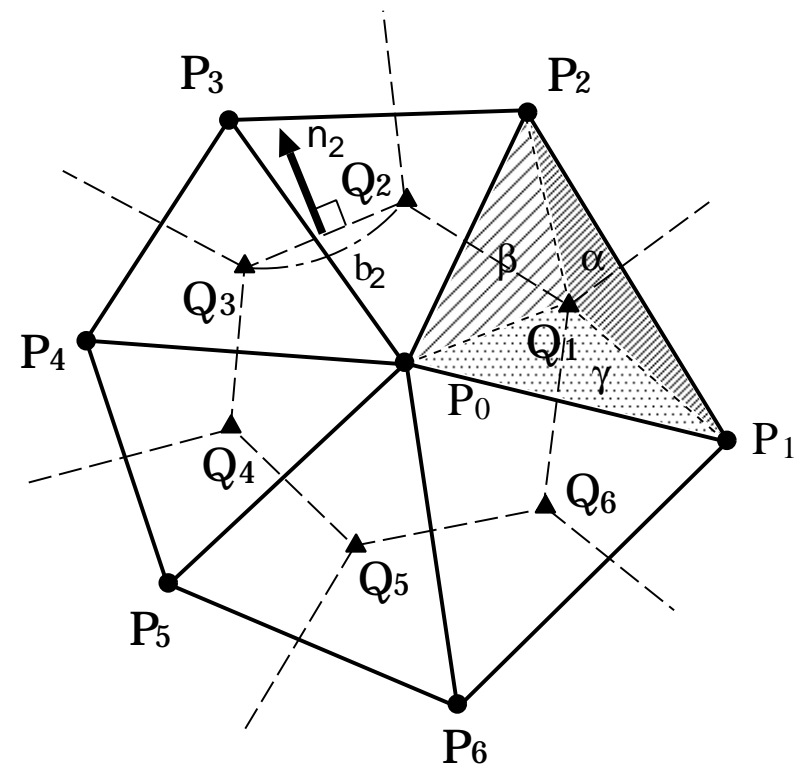

Fig. 7. Horizontal control volume and arrangement of points in case of a pentagonal shape.

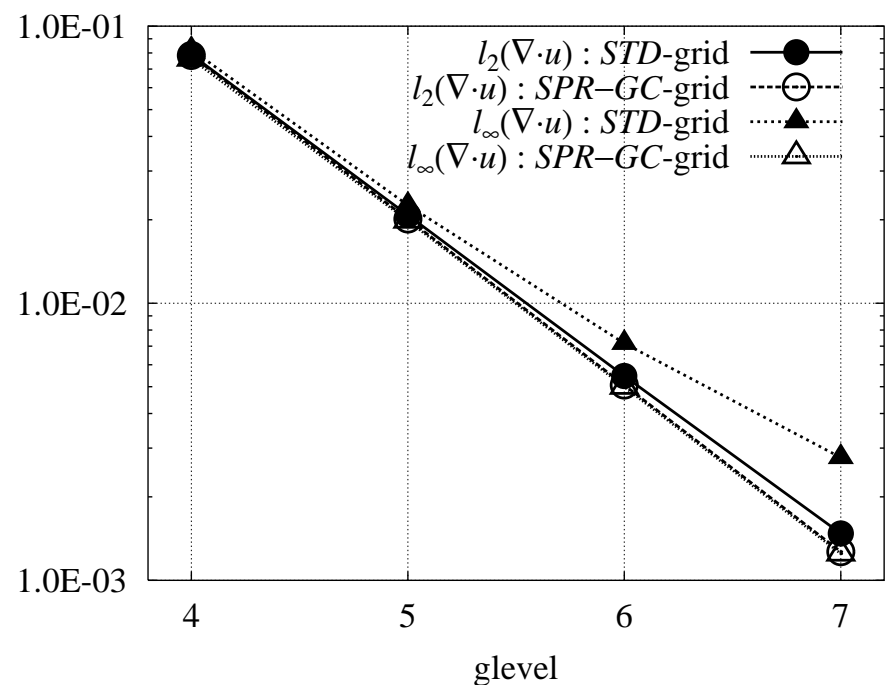

Fig. 8. Convergence properties of error norms for divergence operator. Figure reproduced from [43].

so that we need four equations to update the velocity vector: $\left(V_{h 1}, V_{h 2}, V_{h 3}, W\right)$. From $\mathbf{V}_{h}$ and $W$, we can construct the three-dimensional vector

$$
\mathbf{v}=\mathbf{v}_{\mathbf{h}}+w \hat{\mathbf{k}}=\frac{\mathbf{V}_{h}}{\rho G^{1 / 2} \gamma^{2}}+\frac{W}{\rho G^{1 / 2} \gamma^{2}} \hat{\mathbf{k}}
$$



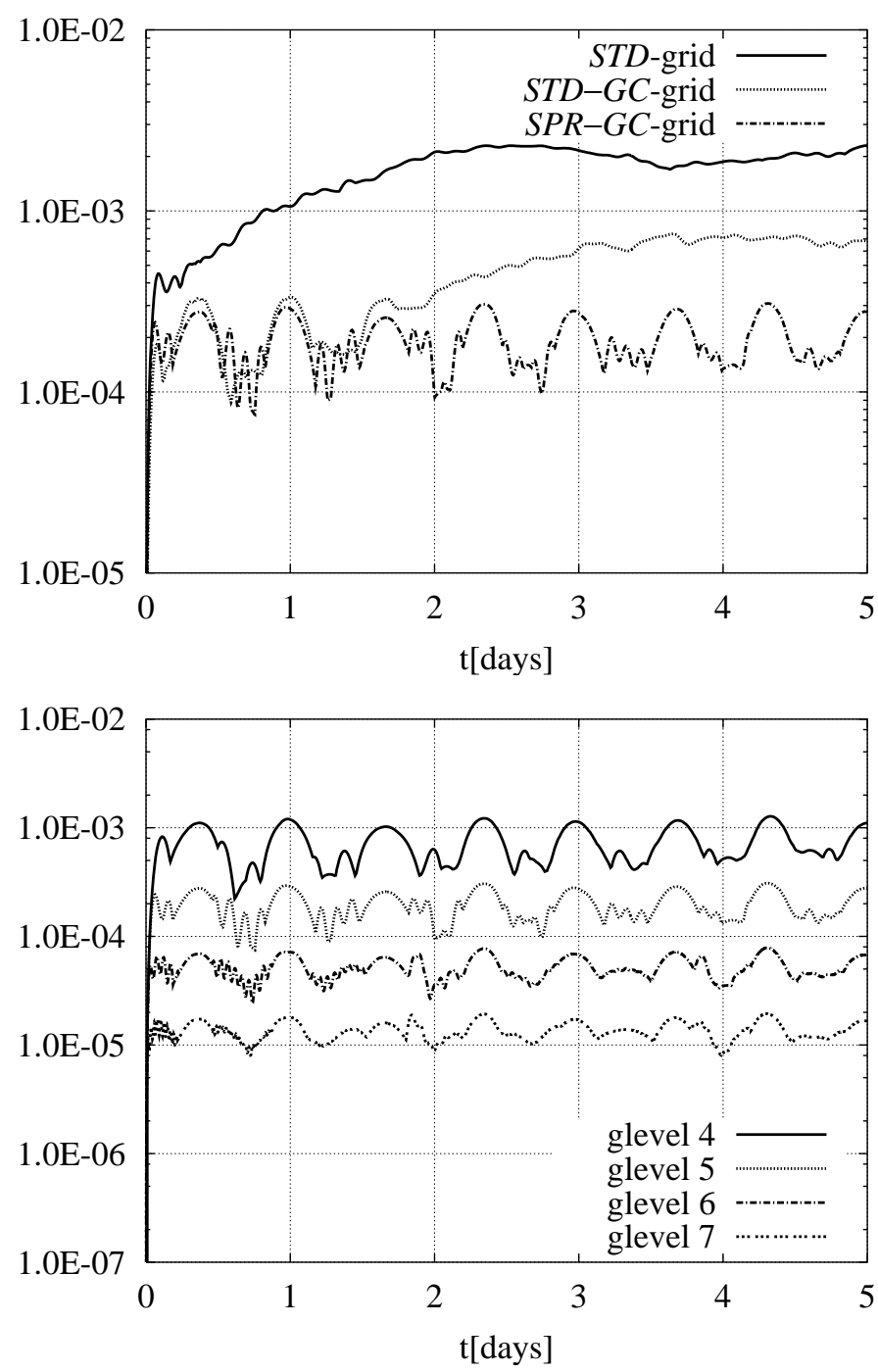

Fig. 9. The time sequence of $l_{\infty}(h)$ norm for Williamson's test case 2. Comparison between the standard grids (STD-grid, STD-GC-grid) and the modified grid (SPR-GC-grid) for glevel-5 (top) and dependency on the resolution from glevel-4 to glevel-7 (bottom). Figure reproduced from [43].

The advection term $\tilde{\mathbf{A}}$ is calculated using (11) by inserting each of the orthogonal components of $\mathbf{v}$ :

$$
\left(v_{1}, v_{2}, v_{3}\right)=\left(v_{h 1}+w \cos \theta \cos \lambda, v_{h 2}+w \cos \theta \sin \lambda, v_{h 3}+w \sin \theta\right),
$$

where $\left(v_{h 1}, v_{h 2}, v_{h 3}\right)=\mathbf{v}_{h} \cdot\left(\mathbf{e}_{1}, \mathbf{e}_{2}, \mathbf{e}_{3}\right)=\left(V_{h 1}, V_{h 2}, V_{h 3}\right) / \rho G^{1 / 2} \gamma^{2}$. The advection vector $\tilde{\mathbf{A}}$ is split again into a horizontal vector as the horizontal advection 
term $\tilde{\mathbf{A}}_{h}$ and a vertical component as the vertical advection term $\tilde{A}_{z}$.

\subsection{Time integration scheme}

In (1)-(4), the left hand side terms are associated with fast propagating waves, while the right hand side terms are related to relatively slow motions. We refer the former to "fast mode terms" and the latter to "slow mode terms". We integrate the prognostic variables with the time-splitting method; that is, the fast mode terms are evaluated at every small time step with a time interval $\Delta \tau$, while the slow mode terms are evaluated at large time steps with a larger time interval $\Delta t$. The split number $N_{s}=\Delta t / \Delta \tau$ is generally 4 .

For the small time step integration, we use the forward-backward scheme based on the HEVI scheme, while we use the Runge-Kutta scheme (either 2nd- or 3rd-order) for the large time step integration. Let a set of prognostic variables at $t=t_{A}$ be known (Fig. 4). We can evaluate the slow mode tendency $S\left(t_{A}\right)$. The fast mode tendency being updated at every small time step, the variables are integrated by using $S\left(t_{A}\right)$ from $t_{A}$ to $t_{B}$. Thus, values of the prognostic variables at $t=t_{B}$ are obtained. Using these values, we can evaluate the slow mode tendency $S\left(t_{B}\right)$. Returning to $t=t_{A}$, the variables are integrated from $t_{A}$ to $t_{C}$ by using $S\left(t_{B}\right)$.

We also introduce the flux division method for the small time step integration [48]; that is, the prognostic variables are further divided into the portions at the current large time step $t\left(t_{A}\right.$ or $t_{B}$ in Fig.4 $)$ and the deviations from them. Let the value of a prognostic variable $\phi$ at the current large time step be $\phi^{t}$ and the deviation from it be $\phi^{*}\left(=\phi-\phi^{t}\right)$. We temporally discretize 
(1)-(4) by expanding the fluxes around the time $t$ :

$$
\begin{aligned}
& \frac{R^{* \tau+\Delta \tau}-R^{* \tau}}{\Delta \tau}+\tilde{\nabla}_{h 0} \cdot \frac{\mathbf{V}_{h}^{*} \tau+\Delta \tau}{\gamma}+\frac{\partial}{\partial \xi}\left(\frac{\mathbf{V}_{h}^{* \tau+\Delta \tau}}{\gamma} \cdot \mathbf{G}^{\mathbf{z}}+\frac{W^{* \tau+\Delta \tau}}{G^{1 / 2}}\right) \\
& =-\left[\tilde{\nabla}_{h 0} \cdot \frac{\mathbf{V}_{h}^{t}}{\gamma}+\frac{\partial}{\partial \xi}\left(\frac{\mathbf{V}_{h}^{t}}{\gamma} \cdot \mathbf{G}^{\mathbf{z}}+\frac{W^{t}}{G^{1 / 2}}\right)\right]+G_{R}^{t}, \\
& \frac{\mathbf{V}_{h}^{* \tau+\Delta \tau}-\mathbf{V}_{h}^{* \tau}}{\Delta \tau}+\tilde{\nabla}_{h 0} \frac{P^{* \tau}}{\gamma}+\frac{\partial}{\partial \xi}\left(\mathbf{G}^{\mathbf{z}} \frac{P^{* \tau}}{\gamma}\right) \\
& =-\left[\tilde{\nabla}_{h 0} \frac{P^{t}}{\gamma}+\frac{\partial}{\partial \xi}\left(\mathbf{G}^{\mathbf{z}} \frac{P^{t}}{\gamma}\right)\right]+G_{\mathbf{V}_{h}}^{t}, \\
& \frac{W^{* \tau+\Delta \tau}-W^{* \tau}}{\Delta \tau}+\gamma^{2} \frac{\partial}{\partial \xi}\left(\frac{P^{* \tau+\Delta \tau}}{G^{1 / 2} \gamma^{2}}\right)+R^{* \tau+\Delta \tau} g \\
& =-\left[\gamma^{2} \frac{\partial}{\partial \xi}\left(\frac{P^{t}}{G^{1 / 2} \gamma^{2}}\right)+R^{t} g\right]+G_{W}^{t}, \\
& \frac{E_{a}^{* \tau+\Delta \tau}-E_{a}^{* \tau}}{\Delta \tau}+\tilde{\nabla}_{h 0} \cdot\left(h_{a}^{t} \frac{\mathbf{V}_{h}^{* \tau+\Delta \tau}}{\gamma}\right) \\
& +\frac{\partial}{\partial \xi}\left[h_{a}^{t}\left(\frac{\mathbf{V}_{h}^{* \tau+\Delta \tau}}{\gamma} \cdot \mathbf{G}^{\mathbf{z}}+\frac{W^{* \tau+\Delta \tau}}{G^{1 / 2}}\right)\right]+\tilde{g}^{t} W^{* \tau+\Delta \tau} \\
& =-\left[\tilde{\nabla}_{h 0} \cdot\left(h_{a}^{t} \frac{\mathbf{V}_{h}^{t}}{\gamma}\right)+\frac{\partial}{\partial \xi}\left[h_{a}^{t}\left(\frac{\mathbf{V}_{h}^{t}}{\gamma} \cdot \mathbf{G}^{\mathbf{z}}+\frac{W^{t}}{G^{1 / 2}}\right)\right]\right] \\
& +\frac{\mathbf{V}_{h}^{t}}{\rho^{t} G^{1 / 2} \gamma^{2}} \cdot\left(\tilde{\nabla}_{h 0} \frac{P^{t}}{\gamma}+\frac{\partial}{\partial \xi}\left(\mathbf{G}^{\mathbf{z}} \frac{P^{t}}{\gamma}\right)\right)-\tilde{g}^{t} W^{t}+G_{E_{a}}^{t},
\end{aligned}
$$

where

$$
\tilde{g}^{t}=g-\frac{1}{\rho^{t} G^{1 / 2} \gamma^{2}}\left[\gamma^{2} \frac{\partial}{\partial \xi}\left(\frac{P^{t}}{G^{1 / 2} \gamma^{2}}\right)+R^{t} g\right] .
$$

In (23), the major part of work done by the pressure gradient force and the buoyancy force is evaluated by using the large time step stratification (24). The enthalpy in the advection terms plays a role of the acoustic wave speed ( $\left.c_{s}^{2}=\left(R_{d} / C_{v}\right) h_{a}^{t}\right)$ and is evaluated at large time steps.

We first integrate (21) by the forward method. Using thus obtained $\mathbf{V}_{h}^{* \tau+\Delta \tau}$, we couple (20), (22), and (23) to construct a one-dimensional Helmholtz equation for $W^{* \tau+\Delta \tau}$ by eliminating $R^{* \tau+\Delta \tau}$ and $P^{* \tau+\Delta \tau}$. To do this, we rewrite 
(23) for the equation of pressure as

$$
\frac{P^{* \tau+\Delta \tau}-P^{* \tau}}{\Delta \tau}+\frac{R_{d}}{C_{v}} \frac{\partial}{\partial \xi}\left[h_{a}^{t}\left(\frac{W^{* \tau+\Delta \tau}}{G^{1 / 2}}\right)\right]+\frac{R_{d}}{C_{v}} \tilde{g}^{t} W^{* \tau+\Delta \tau}=G_{P}
$$

where use is made of the approximate relation between $P^{*}$ and $E_{a}^{*}$ :

$$
E_{a}^{*} \approx \frac{C_{v}}{R_{d}} P^{*}
$$

Note that this relation is exact only in the dry case, and conventionally is a good approximation in this solver [49]. The tendency term $G_{P}$ is the sum of the remaining terms in (23) multiplied by $R_{d} / C_{v}$. After solving the onedimensional Helmholtz solver for $W^{* \tau+\Delta \tau}$, we obtain $R^{* \tau+\Delta \tau}$ from (20). Since (20) is in the flux form, the total integral of mass over the domain is conserved in this scheme.

For the evaluation of internal energy $E^{* \tau+\Delta \tau}$, we use the conservative method proposed by $[48,49]$ to guarantee the conservation of total energy. That is, we use the following flux form of the total energy equation:

$$
\begin{aligned}
& \frac{E_{t o t}^{\tau+\Delta \tau}-E_{t o t}^{\tau}}{\Delta \tau}+\tilde{\nabla}_{h 0} \cdot\left[\left(h_{a}+\phi+k\right)^{t} \frac{\mathbf{V}_{h}^{* \tau+\Delta \tau}}{\gamma}\right] \\
+ & \frac{\partial}{\partial \xi}\left[\left(h_{a}+\phi+k\right)^{t}\left(\frac{\mathbf{V}_{h}^{*} \tau+\Delta \tau}{\gamma} \cdot \mathbf{G}^{\mathbf{z}}+\frac{W^{* \tau+\Delta \tau}}{G^{1 / 2}}\right)\right]=G_{E_{a}+K+\Phi}^{t},
\end{aligned}
$$

where $E_{t o t}=E_{a}+K+\Phi$. Since $(K+\Phi)^{\tau+\Delta \tau}$ is known, $E_{a}^{* \tau+\Delta \tau}$ can be obtained by

$$
E_{a}^{* \tau+\Delta \tau}=E_{a}^{\tau+\Delta \tau}-E_{a}^{t}=E_{t o t}^{\tau+\Delta \tau}-(K+\Phi)^{\tau+\Delta \tau}-E_{a}^{t} .
$$

In the flux form equations, vertical transports of momentum and energy in addition to water mass associated with precipitable water components are 
taken into account. These transports are calculated using an accurate onedimensional conservative semi-Lagrangian scheme [69]. The associated energy transformation from potential energy of precipitating substances to internal energy are properly evaluated after [70].

\subsection{Numerical filters}

For numerical stabilization, we introduce two kinds of numerical filters to the model: divergence damping [71] and biharmonic horizontal diffusion. These filters are somewhat different from our previous report [50].

The divergence damping term is used in the momentum equation to suppress instability associated with the time splitting scheme. Different from the widelyused divergence damping [50, 71], we use the higher order scheme to suppress small scale numerical disturbances. The pressure gradient terms in (2) and (3) are modified as

$$
\begin{aligned}
\tilde{\nabla}_{h 0} \frac{P}{\gamma} & \rightarrow \tilde{\nabla}_{h 0} \frac{P-A_{d H} \tilde{\nabla}^{2} D}{\gamma}, \\
\gamma^{2} \frac{\partial}{\partial \xi}\left(\frac{P}{G^{1 / 2} \gamma^{2}}\right) & \rightarrow \gamma^{2} \frac{\partial}{\partial \xi}\left(\frac{P-A_{d V} \tilde{\nabla}^{2} D}{G^{1 / 2} \gamma^{2}}\right),
\end{aligned}
$$

where $D$ is the three-dimensional divergence of momentum defined by

$$
D=\tilde{\nabla}_{h 0} \cdot \frac{\mathbf{V}_{h}}{\gamma}+\frac{\partial}{\partial \xi}\left(\frac{\mathbf{V}_{h}}{\gamma} \cdot \mathbf{G}^{\mathbf{z}}+\frac{W}{G^{1 / 2}}\right) .
$$

$A_{d H}$ and $A_{d V}$ are the damping coefficients in the horizontal and vertical directions, respectively. They are expressed by using the non-dimensional numbers $\alpha_{d H}$ and $\alpha_{d V}$ : 
$A_{d H}=\alpha_{d H} \frac{\overline{\Delta x}^{4}}{\Delta t}, \quad A_{d V}=\alpha_{d V} \frac{\overline{\Delta x}^{4}}{\Delta t}, \quad$ where $\overline{\Delta x}=\sqrt{\frac{4 \pi r_{0}^{2}}{N_{g}}}$.

$\overline{\Delta x}$ is the mean grid interval in the horizontal directions. The divergence damping term is evaluated every small time step, so that it is integrated by the Euler method with the small time step interval $\Delta \tau$. If the horizontal grid interval is much larger than the vertical one, the same value cannot be used both for the horizontal and vertical directions. In this case, we do not use the vertical divergence damping: $\alpha_{d V}=0$.

The biharmonic horizontal diffusion terms are applied to all the prognostic equations (1)-(4). We do not use numerical diffusions in the vertical direction in general. That is,

$$
[\text { R.H.S. of }(1)-(4)] \rightarrow[\text { R.H.S. of }(1)-(4)]-\tilde{\nabla}_{h 0}^{2}\left[\mu K_{H}\left(\tilde{\nabla}_{h}^{2} \phi\right)\right]
$$

where $(\phi, \mu) \in\left[\left(\rho, G^{1 / 2} \gamma^{2}\right),\left(e, \rho G^{1 / 2} \gamma^{2}\right),\left(\mathbf{v}_{h}, \rho G^{1 / 2} \gamma^{2}\right),\left(w, \rho G^{1 / 2} \gamma^{2}\right)\right] ; K_{H}$ is the lateral diffusion coefficient on $\xi$-surfaces. The diffusion terms are evaluated at every large time step. Using the large time step interval $\Delta t$, the diffusion coefficient is expressed as

$$
K_{H}=\gamma_{H} \frac{\overline{\Delta x}^{4}}{\Delta t}
$$

where $\gamma_{H}$ is the nondimensional diffusion coefficient.

The corresponding damping times for $A_{d H}$ and $K_{H}$ are defined as

$$
\tau_{d i v} \equiv \frac{\overline{\Delta x}^{4}}{A_{d H}}=\frac{\Delta t}{\alpha_{d H}}, \quad \tau_{d i f} \equiv \frac{\overline{\Delta x}^{4}}{K_{H}}=\frac{\Delta t}{\gamma_{H}}
$$

respectively. In general, as the grid interval is halved $\overline{\Delta x} \rightarrow \overline{\Delta x} / 2$ (glevel$n \rightarrow n+1)$, the above diffusion times are halved to suppress smaller scale 
disturbances. We generally set the large time step interval $\Delta t \rightarrow \Delta t / 2$ to keep the numerical stability as glevel is increased $n \rightarrow n+1$. Thus, the nondimensional coefficients $\alpha_{d H}$ and $\gamma_{H}$ are kept constant in general. As default values, we use $A_{d H}=K_{H}=10^{16} \mathrm{~m}^{4} \mathrm{~s}^{-1}$ at the resolution $\overline{\Delta x} \approx 240 \mathrm{~km}$ (glevel-5) and $\Delta t=1200$ sec.

\subsection{Consistency with continuity}

The advection term of a tracer $q$ including water substances is calculated at large time steps using (5). It is pointed out that the consistency with continuity (CWC) for the integration of a tracer should be guaranteed $[72,73]$; that is, the advection of a tracer should be consistently constructed from the equation of density (1). As shown below, CWC is achieved in NICAM by using the time averaged mass flux over small time steps for calculation of advection of tracers.

By omitting the density change due to precipitation fluxes $G_{R}$, the time discretized form of (1) is abbreviated as

$$
\frac{R^{\tau+\Delta \tau}-R^{\tau}}{\Delta \tau}=-\nabla \cdot \boldsymbol{V}^{\tau}
$$

where $R=\rho^{\prime} G^{1 / 2} \gamma^{2}$ and $\boldsymbol{V}^{\tau}$ is the three-dimensional mass flux vector represented in the second and the third terms on the left hand side of (1). In this equation, $R$ is updated at every small time step $\tau$. This means that the density change in the long time step interval $\Delta t$ is given by the average mass flux over $\Delta t$ defined by 


$$
\overline{\boldsymbol{V}}^{t}=\frac{1}{N_{s}} \sum_{n=1}^{N_{s}} \boldsymbol{V}^{t+n \Delta \tau} .
$$

Then, if the tracer per unit volume $Q=\rho G^{1 / 2} \gamma^{2} q$ is integrated as

$$
\frac{Q^{t+\Delta t}-Q^{t}}{\Delta t}=-\nabla \cdot\left(\overline{\boldsymbol{V}}^{t} q^{t}\right)
$$

then (30) with $q=1$ is equal to time average of (29) over $\Delta t$; that it, the consistency with continuity is guaranteed.

In the system with precipitating water substances, the concentration of tracer is further changed by the mass change due to precipitation (6). If an ideal passive tracer $q$ is uniformly given to the initial field, $q=1, q$ becomes larger than 1 in the regions where precipitation occurs. If one wants to keep uniform distribution $q=1$, the effect of precipitation can be calculated by adding the following term on the right hand side of (30):

$$
G_{Q}=-\sum_{n} \frac{\partial}{\partial \xi}\left(q \frac{W_{n}^{*}}{G^{1 / 2}}\right) .
$$

In fact, the effects of precipitation depend on properties of tracers, so that the form of (31) may be different between tracers. In summary, in nonhydrostatic models with precipitating substances, in order to keep the uniform distribution of tracer, the mass flux should be consistent with continuity equation (CWC), and the mass change due to precipitation should be properly counted.

\section{Numerical results}

Various experiments are already done using NICAM. Several dynamical core experiments are shown in [50], and high-resolution dry experiments for extra- 
tropical cyclones, together with consistency of horizontal and vertical grid intervals, are described by [51]. "Global cloud resolving experiments" using 3.5 km-horizontal-mesh interval (glevel-11) under idealized aqua-planet condition [55] is reported by $[8,52,54]$. Climate sensitivity with using $7 \mathrm{~km}$-mesh grid is examined by [53]. Instead of cumulus parameterization used in heuristic AGCMs, a cloud microphysical scheme [74] is used for these $3.5 \mathrm{~km}$ - and 7 km-mesh experiments.

The $3.5 \mathrm{~km}$-mesh aqua-planet experiment is performed for 10 days after spinup runs using coarser resolution $14 \mathrm{~km}$ and $7 \mathrm{~km}-\mathrm{mesh}$, as described in [52]. The time steps of the 3.5, 7, $14 \mathrm{~km}$-mesh experiments are 15, 30, and $30 \mathrm{~s}$, respectively [8]. Figure 10 shows a global view of the outgoing longwave radiation (OLR) of the $3.5 \mathrm{~km}$-mesh experiment. This is 90 min average between 0:00-1:30 at 5th day from the integration. This shows a hierarchical structure of tropical convection: meso-scale circulation associated with individual deep clouds, cloud clusters, super-cloud clusters, and planetary scale convectively coupled Kelvin waves. Figure 11 shows a Hovmöller diagram of precipitation averaged over latitudes $2^{\circ} \mathrm{N}-2^{\circ} \mathrm{S}$ for the 3.5 and $7 \mathrm{~km}$-mesh experiments. Note that the $3.5 \mathrm{~km}$-mesh run studied by $[52,54]$ corresponds to the time duration between 80 and 90 days, and that Fig.10 is for day 85. As seen by diagonal signals in Fig. 11, super cloud clusters propagates eastward; these have a Kelvin wave structure in dynamical fields [54]. Inside the super cloud clusters, cloud clusters with size of a few hundred $\mathrm{km}$ are moving westward (Fig. 11, right bottom). They are persistently seen within the super cloud clusters during the integration. This multi-scale structure and motions of cloud systems possess similar characteristics seen in observations $[75,76]$.

The above experiment is conducted under the same experimental condition 
proposed by [55], using the CTL (control) case. Many of research groups conducted this experiment using different AGCMs. Mainly because of the difference in cumulus closures, the resulting signal of cloud systems were very different between AGCMs [77]; Reading); some results show westward propagation of large scale organized convective systems, and others show scattered signals. To reproduce realistic eastward propagation of cloud systems seen in the observations, cumulus closures must be properly tuned. Our results with the global cloud resolving simulations will give a guidance to the choice of cumulus closures in AGCMs.

Next, a preliminary result of a global cloud resolving simulation under realistic land and ocean distribution with topography is shown. Detailed analysis of this experiment is reported in [78]. Physics package is the same as that used in [52], except for the effect of moisture is taken into account for the calculation of Richardson number of the boundary layer process [79]. A simple "bucket" scheme is used for the land process and the sea surface temperature is prescribed; the bucket scheme assumes that at each of grid point over land maximum of water content is restricted by a specified value of bucket height. Giving a NCAR/NCEP reanalysis data at 00 UTC 1 Apr.2004 as an initial condition, a $3.5 \mathrm{~km}$-mesh global simulation is conducted for a week. Although the initial field is coarse resolution $\left(1^{\circ} \times 1^{\circ}\right)$, cloud systems are spun up within a few hours. Figure 12 shows global view of OLR at 00 UTC 5 Apr. of the numerical simulation. The results show that the typhoon (T0401, SUDAL) is realistically simulated over the western Pacific, though the organization of convective system seems a little too strong. Cloud patterns of mid-latitude extra-tropical cyclones are well captured. Different from regional models in which side boundary data are prescribed, large scale circulations are to be 
spontaneously reproduced in global cloud resolving simulations. We are investigating climatology of model results by performing longer simulation.

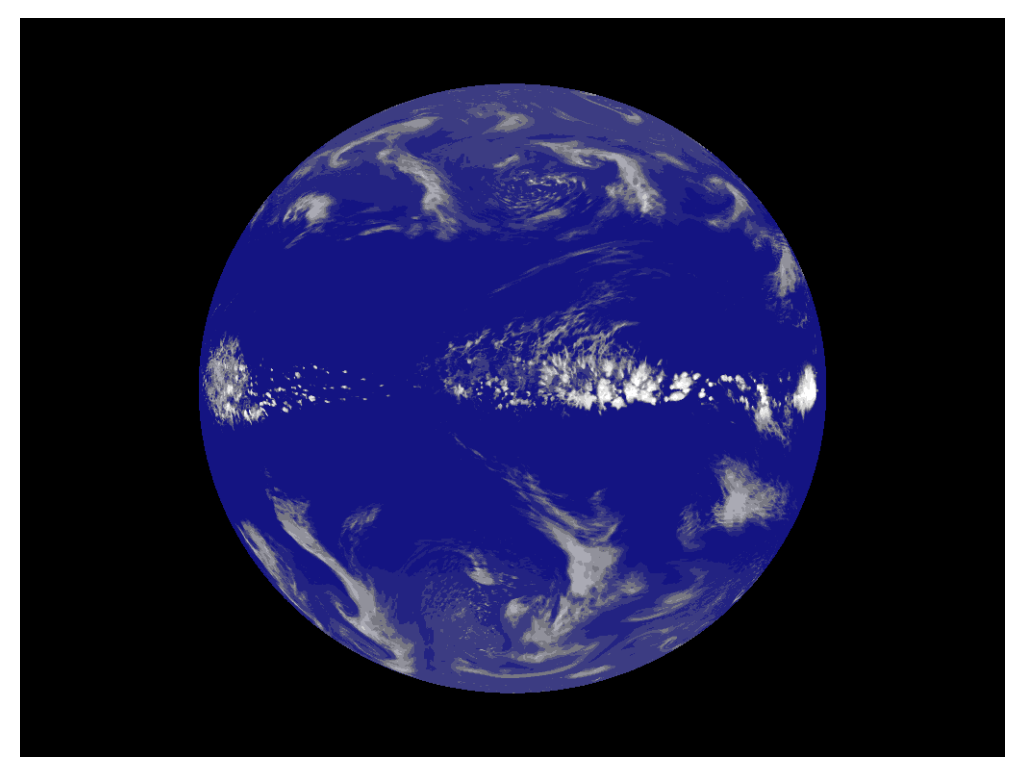

Fig. 10. Global view of the outgoing longwave radiation (OLR) produced by NICAM with the $3.5 \mathrm{~km}$-mesh aqua-planet experiment.
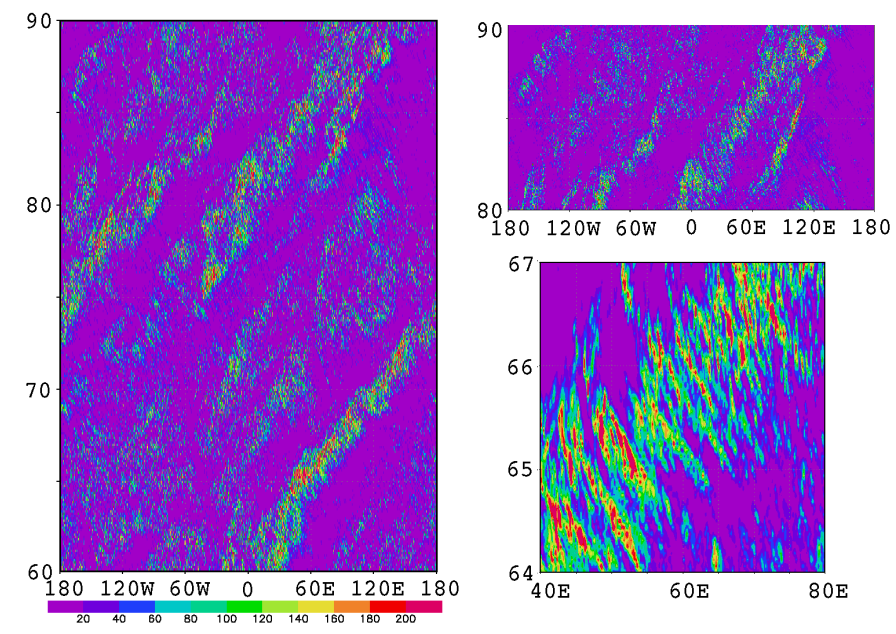

Fig. 11. Hovmöller diagrams of precipitation along the equator averaged in latitudes $2^{\circ} \mathrm{N}-2^{\circ} \mathrm{S}$. (left) $7 \mathrm{~km}-\mathrm{mesh}$ run, (right top) $3.5 \mathrm{~km}-m e s h$ run, and (right bottom) zoomed up in the region days 64-67 and latitudes 40E-80E for $7 \mathrm{~km}-\mathrm{mesh}$ run. Unit is $m m$ day $^{-1}$. After [8]. 


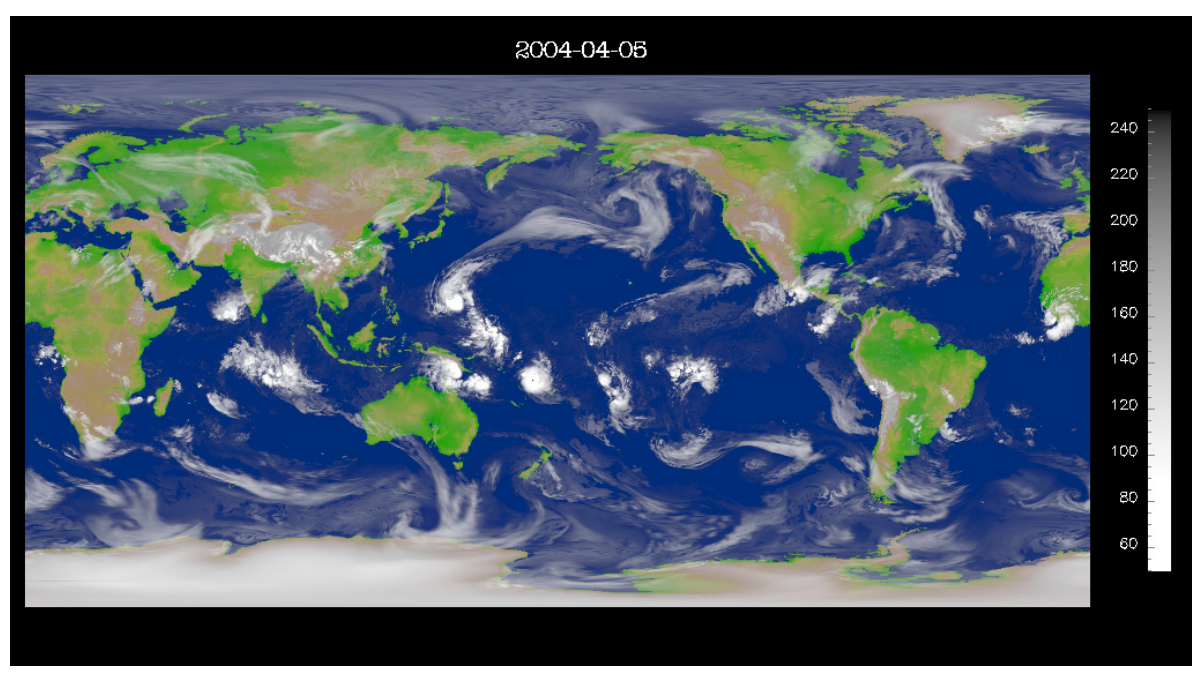

Fig. 12. Global view of OLR produced by NICAM with the $3.5 \mathrm{~km}$-mesh realistic land and sea distribution experiment for 00 UTC 5 Apr. 2004. Integration is started from 00 UTC 1 Apr. 2004.

\section{Future perspective}

Before we discuss the future perspective, we shall briefly retrospect to explain the origin of the project. The background and motivation of this undertaking to develop an ultra-high resolution model is to make a quantum leap in progress in global atmospheric modeling by fully exploiting the grand computing power of the Earth Simulator (ES) whose development began nearly the same time of the foundation of the Frontier Research Center for Global Change (FRCGC) as a closely related project.

From a crude estimate, the highest horizontal resolution of global atmospheric model practically possible by use the originally planned performance of the ES turned out to be $10 \mathrm{~km}$. Actually a model of this resolution was realized soon after the completion of the ES simply by increasing the horizontal resolution of an already existing AGCM [1]. However at the FRCGC, we considered that a $10 \mathrm{~km}$ mesh AGCM with hydrostatic approximation accompanied by 
convection parameterization would not be a good target as a new AGCM to be run on the ES because no essentially new mechanism can be included in it and hence qualitatively new advance was not expected. In contrast to this, if we can express deep convective clouds in the tropics directly by the grid system without parameterization, global atmospheric modeling will make a quantum leap. It is known that cumulonimbus clouds in the tropics are the manifestation of "vertical convection", that is they transport energy from the surface of the earth to the upper troposphere to compensate the radiative energy imbalance. Since the convection caused by the vertical differential heating is a fundamental process as well as the convection caused by the latitudinal differential heating which appear as extra-tropical cyclones (and anticyclones) and was the first target of AGCMs [80], we believe that "general circulation" models are much more complete when both these fundamental processes are directly represented. Further the vertical convection occurs in the form of cloud clusters or mesoscale convective systems (MCSs) as its elementary structure which is unique to the earth's atmosphere being different from Bénard convection widely seen in nature [81]. Perhaps for this uniqueness and their self-sustaining mechanism, cloud clusters behave more or less autonomously thus making "parameterization" difficult so that direct simulation of them as a component of the global circulation seems to be a challenging target. On the basis of these considerations, we decided to develop "mesoscale convective system resolving" AGCM as one of the major scientific objectives of the development of the ES [82].

However, a serious difficulty was foreseen at the beginning. As mentioned previously, the highest resolution of AGCMs practically possible on the ES was estimated about $10 \mathrm{~km}$ whereas the size of updraft region of mesoscale convec- 
tive systems is $10-20 \mathrm{~km}$ in most cases so that the horizontal grid size needed to express the systems was estimated to be less than $5 \mathrm{~km}$. Since the computer time required for time integrations of $5 \mathrm{~km}$ mesh model is about 10 times longer than that for $10 \mathrm{~km}$ mesh, the model might not be used for long term simulations of practical purposes. Notwithstanding this situation, we initiated the model development believing that global cloud system resolving model will become necessary in the near future and our undertaking would become a useful first step toward it. Fortunately, the actual computational performance of the ES turned out to be about 3 times faster than originally declared target of the ES development. This was the result of the well-considered computer architecture of the vector-parallel processors which worked beyond expectation and of the efficient numerical codes based on the icosahedral geometry. Hence, NICAM has been producing scientifically interesting results with the $3.5 \mathrm{~km}-\mathrm{mesh}$ as described in the preceding section.

As described above, the primary objective of developing NICAM is to simulate mesoscale convective cloud systems in the tropics. Of course, with such an ultra-high resolution NICAM can be applied to a wide class of problems. An example already underway is numerical experimentation on clouds' feedback effects on global warming [53] in which direct calculation of small-scale clouds will give us more reliable results on changes of cloud amount and reflectivity in a warm world. Further development toward application to the nextgeneration numerical weather prediction is also under consideration. Here, however, we shall confine our discussion to the future direction of research on tropical atmosphere. As already noted, we can say that numerical simulation of the atmospheric general circulation becomes more reliable by explicit treatment of the convection in the tropics. In other words, Phillips' work [80] and 
subsequent AGCM experiments were basically those on the middle and high latitude circulations where baroclinic instability due to the latitudinal temperature gradient is the primary process. The size of the "energy-containing eddies" or the most unstable wave length is $O\left(10^{6}\right) \mathrm{m}$, whereupon numerical models with a grid size of $O\left(10^{5}\right) \mathrm{m}$ could simulate well the structure and circulations of the atmosphere in the middle-high latitude regions. It is noted that with an increase of resolution finer structures such as sharpening of fronts, spiral winding-up of vortices and so on, become better simulated quite naturally because these smaller scale structures are produced through nonlinear processes (downscale cascade) from major energy bearing baroclinic vortices. It is also noted that the atmosphere remains stably stratified; even though condensation takes place, the associated latent heat release does not destroy the mean stability.

In contrast, the characteristics of the tropical atmospheric dynamics are quite different from those of middle-high latitudes. The tropical atmosphere is in a state of the latent instability; namely the equivalent potential temperature near the surface is generally higher than those at upper levels and deep cumulonimbus can develop almost everywhere in most periods of the year. Instead, the latitudinal temperature gradient is small and baroclinic instability cannot occur. Thus the primary energy source for motions in the tropics lies in the vertical convection which appears as MCSs as mentioned previously. They have a definite structure which enables vertical overturning in a state of the latent instability (e.g., [83]). Convections of this relatively small scale (100-300 km) unit structure occur in various ways. Occasionally they produce larger-scale aggregates and associated circulation systems through mechanisms yet to be understood. Once we have completed the model to resolve the el- 
ementary structure of convection, we will be able to understand dynamical (and cloud physical) mechanisms working in various form of occurrences of mesoscale convective systems (MCSs) and related phenomena. We are aware that it is a huge step from being able to resolve the structure of convection to actually understanding dynamical mechanisms. But we will be in a position to do numerical experiments that should advance our understanding more rapidly. Unlike baroclinic unstable waves in the middle-high latitudes, analytical methods (e.g., linear instability theory) are not applicable and moreover detailed observations are not easy for tropical convections.

At present, we recognize the following four forms as modes of the occurrence of MCSs to be immediate objects of research.

(1) MCSs generated by land-sea heating contrast synchronized with the diurnal cycle. Though phenomenon like "afternoon shower" is very common and has been known from ancient times their structure and evolution as MCSs have become clear rather recently pioneered by [84]. It is now known that this type of precipitation dominates over the maritime continent-Indian ocean region (e.g., [85]).

(2) MCSs forming big aggregates and associated with large-scale circulation systems. Phenomena of this type is called either "super cloud cluster" $[75,86]$ or "convectively coupled equatorial waves" and their existence has come to be clear quite recently [87-89]. This mode of the occurrence of MCSs are already reproduced by NICAM in the aqua-planet experiment [52], so that they are the most natural form of larger-scale structure resulting from MCSs. We may regard this as the tropical counterpart of extra-tropical cyclones (baroclinic waves) because both of these appear spontaneously in idealized numerical experiments $[52,80]$. 
(3) Madden-Julian Oscillation (MJO) or Tropical Intra Seasonal Oscillation (TISO). This is the most pronounced and largest scale phenomenon consisting from MCSs and accompanied by the particular circulation system. Since its discovery $[90,91]$ has been investigated by many researchers because of its important role in global weather and climate. However, the mechanisms of its generation, maintenance and variation are not yet completely understood. And the lack of simulations with acceptable fidelity of the phenomenon in numerical models is a hardest barrier for weather prediction and climate simulation (e.g., [92]).

(4) MCSs embedded in tropical cyclones and easterly waves (e.g., [93]). . This was the first object of studies on interaction between convection and largescale circulation systems from which the concept of CISK (Conditional Instability of the Second Kind [94]) and "parameterization of convection" emerged. Naturally the parameterization works reasonably well in this case. It is noted that these weather/circulation systems posses clear vorticity fields and the conservative nature of vorticity ensures their identity and sustainability. It is interesting that these phenomena were not found in the aqua-planet experiment by use of NICAM but appeared in the experiments under realistic conditions implying that "mother vorticity" associated with the shear zone of off-equatorial ITCZ (the Intertropical Convergence Zone) or monsoon trough are necessary for their generation

As described above MCSs in the tropics produce a variety of weather / circulation systems but their mechanisms are much less understood compared with those at middle-high latitudes.

Summing up the foregoing discussions, we can say that the atmosphere consists from two different worlds when viewed from the nature of their dynamics 
(Fig. 13). One is the world at middle-high latitudes where the atmosphere is stably stratified and extra-tropical cyclones and anticyclones generated by baroclinic instability dominate. The dynamics in this world is basically governed by the potential vorticity (PV) conservation and because of this clear and well-defined basis. Understanding of various phenomena and their modeling are now matured including numerical weather prediction. We may call this "quasi-2-D Vortex world" or "PV world". On the contrary in the second world at low latitudes the primary energy source lies in individual convections (MCSs) and modes of their occurrence are diverse as explained previously. Some of them appear to be coupled with large-scale waves but not associated with vorticity except for mode (4) in the aforementioned classification. Thus we may call "convection/wave world", or "VP world". Here VP stands for velocity potential. Though VP is not a physical or dynamical entity as PV, VP is widely used to depict various circulations such as Hadley, Walker, monsoon circulations and also MJO, and may be suited to symbolize this world. As already mentioned understanding of the dynamics and numerical modeling of this world are still at infant stage. Cloud system resolving AGCMs like NICAM will become a powerful research tool for understanding of those phenomena in the tropics and will contribute to the progress of weather and climate predictions.

\section{Summary}

A new type of the atmospheric general circulation model based on the nonhydrostatic system and the icosahedral grid is developed. The new model is called Nonhydrostatic ICosahedral Atmospheric Model (NICAM). Details of the gov- 

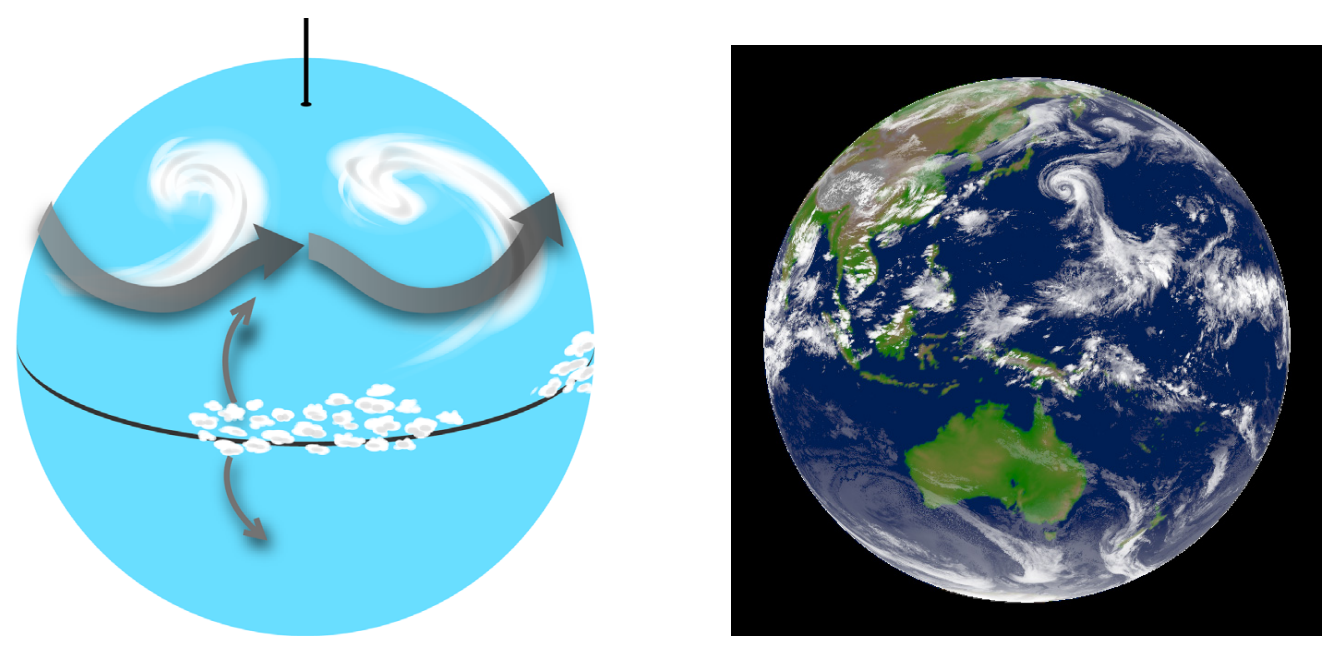

Fig. 13. Pictures to help grasping the notion of "Two-worlds" of the atmospheric general circulation. The left panel is a schematic picture based on result of the aquaplanet experiment and the right panel is an IR image of clouds of the real earth taken by MTSAT-1R on 9 UTC 4 Sep. 2006. Because of near summer conditions in the northern hemisphere, extra-tropical cyclones are not seen clearly there but the southern hemisphere is covered by smooth pattern of large-scale weather systems in contrast to the tropics where smaller-scale convective clouds of various forms are distributed.

erning equations with moist processes and outlines of numerical procedures are described in the present paper. An optimal icosahedral grid is constructed using spring dynamics to achieve higher numerical accuracy. The nonhydrostatic equations are discretized in the flux form using the finite volume method over the modified icosahedral grid. The numerical scheme guarantees conservation of mass, water, and total energy, so that NICAM is suitable for long term integration. The consistency with continuity (CWC) for tracer components is also guaranteed.

Numerical simulations with $3.5 \mathrm{~km}$-horizontal-mesh on the globe are performed over idealized aqua-planet condition and realistic land and sea distribution using the Earth Simulator. The aqua-planet experiment shows multi-scale 
structure of tropical convection; super cloud clusters propagate eastward with phase speed similar to the observations and cloud clusters move westward within super cloud clusters. This hierarchical structure of cloud systems is very similar to the observed one. Preliminary results of a more realistic experiment with land and sea distribution show organization of cloud systems such as typhoons.

We argued future perspective of the use of the global cloud resolving models for studying meso-scale convective systems in the tropics. We will examine results in various perspectives using observational data, particularly satellite data. Such an approach is taken by [95] using the aqua planet experiment data of the $3.5 \mathrm{~km}$-mesh experiment. In such a new era, more efforts toward fusion of satellite observations and ultra-high resolution modeling should be devoted.

\section{Acknowledgments}

Numerical experiments of NICAM were done using the Earth Simulator. This research was supported by Core Research for Evolutional Science and Technology, Japan Science and Technology Agency (CREST, JST). We thank Prof. Tamio Takamura of Chiba Univ. and Prof. Hirohiko Ishikawa of Kyoto Univ. for supports in producing the MTSAT-1R image. We also thank careful reading by reviewers and the editor, Prof. Piotr Smolarkiewicz. 


\section{A Operator notation}

We summarize notations of the differential operators in this appendix. In order to describe the governing equations separately in the horizontal and vertical directions, we divide terms of the differential operators into horizontal and vertical components. The three-dimensional gradient operator for a scalar $\varphi$ and the three-dimensional divergence operator for a vector $\mathbf{u}$ are respectively written as

$$
\begin{aligned}
\nabla \varphi & =\nabla_{h} \varphi+\hat{\mathbf{k}} \frac{\partial \varphi}{\partial z}=\frac{\hat{\mathbf{i}}}{r \cos \theta} \frac{\partial \varphi}{\partial \lambda}+\frac{\hat{\mathbf{j}}}{r} \frac{\partial \varphi}{\partial \theta}+\hat{\mathbf{k}} \frac{\partial \varphi}{\partial z} \\
\nabla \cdot \mathbf{u} & =\nabla_{h} \cdot \mathbf{u}_{h}+\frac{1}{r^{2}} \frac{\partial\left(r^{2} u_{z}\right)}{\partial z} \\
& =\frac{1}{r \cos \theta}\left[\frac{\partial u_{\lambda}}{\partial \lambda}+\frac{\partial\left(u_{\theta} \cos \theta\right)}{\partial \theta}\right]+\frac{1}{r^{2}} \frac{\partial\left(r^{2} u_{z}\right)}{\partial z}
\end{aligned}
$$

where $\nabla_{h}$ is the horizontal gradient operator, $\hat{\mathbf{i}}$ and $\hat{\mathbf{j}}$ are unit vectors in the longitudinal and latitudinal directions, respectively; and $\hat{\mathbf{k}}$ is outward unit vector in the vertical direction. $\theta$ and $\lambda$ are latitude and longitude, respec-

tively, A vector $\mathbf{u}$ is decomposed of $u_{z}(=\mathbf{u} \cdot \hat{\mathbf{k}})$ and $\mathbf{u}_{h}\left(=\mathbf{u}-\hat{\mathbf{k}} u_{z}\right)$, and $u_{\lambda}$ and $u_{\theta}$ are the longitudinal and latitudinal components, respectively. $z$ is the vertical coordinate, defined by $z \equiv r-r_{0}$, where $r_{0}$ is the earth radius. We do not consider ellipsoid of the earth shape, and assume that the radius at the sea level is constant; hence $z$ is height from the sea level. Hereafter, for an arbitrary three dimensional vector, a bold character with subscript $h$ denotes the horizontal vector component and italic character with subscript $z$ denotes the vertical component. $\nabla_{h}$. is the spherical divergence operator.

Standardly, we use the deep atmosphere formulation. For the shallow atmosphere approximation over a spherical earth, $r$ in (A.1) and (A.2) are replaced 
by a constant $r_{0}$. To enable the optionality of the deep and shallow approximations, we introduce a factor $\gamma \equiv r / r_{0}$. With this, we readily derive the following relations:

$$
\gamma \nabla_{h} \varphi=\nabla_{h 0} \varphi, \quad \gamma \nabla_{h} \cdot \mathbf{u}=\nabla_{h 0} \cdot \mathbf{u}
$$

where

$$
\begin{aligned}
\nabla_{h 0} \varphi & =\frac{\hat{\mathbf{i}}}{r_{0} \cos \theta} \frac{\partial \varphi}{\partial \lambda}+\frac{\hat{\mathbf{j}}}{r_{0}} \frac{\partial \varphi}{\partial \theta}, \\
\nabla_{h 0} \cdot \mathbf{u}_{h} & =\frac{1}{r_{0} \cos \theta}\left[\frac{\partial u_{\lambda}}{\partial \lambda}+\frac{\partial\left(u_{\theta} \cos \theta\right)}{\partial \theta}\right] .
\end{aligned}
$$

To include the topographical effects, we introduce the terrain-following coordinates with a transformed vertical coordinate $\xi$ - an arbitrary monotonic function of height $[96,97]$. In the current model, we simply use a linear relation between $z$ and $\xi$ :

$$
\xi=\frac{z_{T}\left(z-z_{s}\right)}{z_{T}-z_{s}}
$$

where $z_{T}$ is the top of the model domain and is constant in the entire globe; $z_{s}$ is the surface height which depends on the horizontal location. Here, we define gradient and divergence operators on a constant $\xi$ shell:

$$
\begin{aligned}
\tilde{\nabla}_{h 0} \varphi & \equiv \frac{\hat{\mathbf{i}}}{r_{0} \cos \theta}\left(\frac{\partial \varphi}{\partial \lambda}\right)_{\xi}+\frac{\hat{\mathbf{j}}}{r_{0}}\left(\frac{\partial \varphi}{\partial \theta}\right)_{\xi}, \\
\tilde{\nabla}_{h 0} \cdot \mathbf{u}_{h} & \equiv \frac{1}{r_{0} \cos \theta}\left[\left(\frac{\partial u_{\lambda}}{\partial \lambda}\right)_{\xi}+\left(\frac{\partial\left(u_{\theta} \cos \theta\right)}{\partial \theta}\right)_{\xi}\right] .
\end{aligned}
$$

Note that these operators are evaluated at the sea level $r_{0}$. We define the metrics as

$$
G^{1 / 2} \equiv\left(\frac{\partial z}{\partial \xi}\right)_{h}, \quad \mathbf{G}^{\mathbf{z}} \equiv \nabla_{h 0} \xi=-\frac{\tilde{\nabla}_{h 0} z}{G^{1 / 2}}
$$


where $(\partial / \partial \xi)_{h}$ denotes a derivative in the vertical direction. Using this, we obtain the following relations:

$$
\begin{aligned}
G^{1 / 2} \nabla_{h 0} \varphi & =\tilde{\nabla}_{h 0}\left(G^{1 / 2} \varphi\right)+\frac{\partial}{\partial \xi}\left(\mathbf{G}^{\mathbf{z}} G^{1 / 2} \varphi\right) \\
G^{1 / 2} \nabla_{h 0} \cdot \mathbf{u}_{h} & =\tilde{\nabla}_{h 0} \cdot\left(G^{1 / 2} \mathbf{u}_{h}\right)+\frac{\partial}{\partial \xi}\left(G^{1 / 2} \mathbf{u}_{h} \cdot \mathbf{G}^{\mathbf{z}}\right) . \\
G^{1 / 2} \frac{\partial \varphi}{\partial z} & =\frac{\partial \varphi}{\partial \xi}
\end{aligned}
$$

Note that the above equations are not satisfied if $\nabla_{h}()$ and $\nabla_{h} \cdot()$ are used instead of $\nabla_{h 0}()$ and $\nabla_{h 0} \cdot()$.

\section{B The basic equations}

\section{Continuity equations}

We consider the system in which unit volume of the air is composed of dry air, water vapor, and liquid and solid phases of water. In this paper, equations are formulated using the one-moment bulk formula for cloud microphysical schemes, where the mass concentrations of water categories are treated as prognostic variables and the numbers of concentration are not. There are both airborne and precipitating components of liquid and solid phases of water, so that these consist of more than or equal to two components, designated by indices $j$ and $k$, respectively. In general, we call airborne component of liquid water "cloud water", and that of solid water "cloud ice". Precipitating components are categorized in many species depending on cloud microphysical schemes. In a simple case, precipitating components of liquid air is "rain", and that of solid water is "snow". If we use more comprehensive scheme such as Lin et al. [98], precipitating components of solid water composed of more categories 
such as "snow", "hail", and "graupel". Up to now, we have implemented cloud schemes of warm rains [27, 99] and cold rains [74, 98, 100].

Let the total density of moist air be denoted by $\rho$, the mass concentration of dry air $q_{d}$, that of vapor $q_{v}$, that of the $j$-th component of liquid water $q_{l, j}$, and that of the $k$-th component of solid water $q_{i, k}$. Total number of liquid water components is $j_{\max }$, and that of solid water components is $k_{\max }$, The continuity equations of dry air, water vapor, liquid and solid components of water are respectively written as

$$
\begin{aligned}
& \frac{\partial\left(\rho q_{d}\right)}{\partial t}+\nabla_{h} \cdot\left(\rho q_{d} \mathbf{v}_{h}\right)+\frac{1}{r^{2}} \frac{\partial\left(r^{2} \rho q_{d} w\right)}{\partial z}=s_{d} \\
& \frac{\partial\left(\rho q_{v}\right)}{\partial t}+\nabla_{h} \cdot\left(\rho q_{v} \mathbf{v}_{h}\right)+\frac{1}{r^{2}} \frac{\partial\left(r^{2} \rho q_{v} w\right)}{\partial z}=s_{v} \\
& \frac{\partial\left(\rho q_{l, j}\right)}{\partial t}+\nabla_{h} \cdot\left(\rho q_{l, j} \mathbf{v}_{h}\right)+\frac{1}{r^{2}} \frac{\partial\left[r^{2} \rho q_{l, j}\left(w+w_{l, j}^{*}\right)\right]}{\partial z}=s_{l, j} \\
& \frac{\partial\left(\rho q_{i, k}\right)}{\partial t}+\nabla_{h} \cdot\left(\rho q_{i, k} \mathbf{v}_{h}\right)+\frac{1}{r^{2}} \frac{\partial\left[r^{2} \rho q_{i, k}\left(w+w_{i, k}^{*}\right)\right]}{\partial z}=s_{i, k}, \\
& \text { for } 1 \leq k \leq k_{\max }
\end{aligned}
$$

where $s_{*}$ represents source terms of each categories including diffusion and conversion between water categories. $\mathbf{v}_{h}$ and $w$ are the horizontal velocity vector and vertical velocity of the air; $w_{l, j}^{*}$ and $w_{i, k}^{*}$ are terminal velocities relative to the air; $t$ is the time. Following Ooyama [70] and Satoh [49], the horizontal velocity is the same as the air irrespective of water categories, while the vertical velocity depends on categories of water, as represented by $w_{l, j}^{*}$ and $w_{i, k}^{*}$. We assume that the sum of the source terms should vanish:

$$
s_{d}+s_{v}+\sum_{j=1}^{j_{\max }} s_{l, j}+\sum_{k=1}^{k_{\max }} s_{i, k}=0
$$

This implies that both the sum of conversion terms between water categories 
and the sum of diffusion terms vanish. The formulations of conversion terms depend on cloud microphysical schemes. We do not describe the explicit form of these terms in this paper, but refer the interested reader to [27, 74, 98-100] for a complete exposition.

It is convenient to use sums of liquid phases of water and solid phases of water. Mass concentrations and source terms are defined by

$$
q_{l}=\sum_{j=1}^{j_{\max }} q_{l, j}, \quad s_{l}=\sum_{j=1}^{j_{\max }} s_{l, j} ; \quad q_{i}=\sum_{k=1}^{k_{\max }} q_{i, k}, \quad s_{i}=\sum_{k=1}^{k_{\max }} s_{i, k} .
$$

The mass concentrations of total water is $q_{w}=q_{v}+q_{l}+q_{i}$ and that of dry air is $q_{d}=1-q_{w}$. Summing (B.1), (B.2), (B.3) over $j$, and (B.4) over $k$, and using (B.5), we obtain the continuity equation of total density as

$$
\begin{aligned}
& \frac{\partial \rho}{\partial t}+\nabla_{h} \cdot\left(\rho \mathbf{v}_{h}\right)+\frac{1}{r^{2}} \frac{\partial\left(r^{2} \rho w\right)}{\partial z} \\
= & -\sum_{j=1}^{j_{\max }} \frac{1}{r^{2}} \frac{\partial\left(r^{2} \rho q_{l, j} w_{l, j}^{*}\right)}{\partial z}-\sum_{k=1}^{k_{\max }} \frac{1}{r^{2}} \frac{\partial\left(r^{2} \rho q_{i, k} w_{i, k}^{*}\right)}{\partial z} .
\end{aligned}
$$

The first and second terms on the right hand side represent flux convergences of liquid and solid water due to precipitating velocities relative to the mass center of the air, respectively.

$\underline{\text { Momentum equation }}$

The momentum equation derives from the fundamental Navier-Stokes' equations, posed in the rotating reference frame with the origin in the center of the Earth (Fig. 1). In a vector notation, the momentum equation for a multiphase atmospheric fluid is compactly written as

$$
\frac{\partial(\rho \mathbf{v})}{\partial t}+\nabla \cdot(\rho \mathbf{v} \otimes \mathbf{v})+2 \rho \boldsymbol{\Omega} \times \mathbf{v}=-\nabla p-\rho g \hat{\mathbf{k}}+\mathbf{f}
$$


$-\sum_{j=1}^{j_{\max }} \nabla \cdot\left(r^{2} \rho q_{l, j} \mathbf{v}_{l, j}^{*} \otimes \mathbf{v}\right)-\sum_{k=1}^{k_{\max }} \nabla \cdot\left(r^{2} \rho q_{i, k} \mathbf{v}_{i, k}^{*} \otimes \mathbf{v}\right)$,

where $p$ is pressure; $g, \boldsymbol{\Omega}$, and $\mathbf{f}$ are the acceleration due to gravity, the angular velocity of the earth, and the frictional force, such as viscosity, respectively; $\otimes$ and $\times$ stand for the tensor product and the outer product, respectively. The last two terms on the right hand side of (B.7) represent the drag forcing, where $\mathbf{v}_{l, j}^{*}=\left(0,0, w_{l, j}^{*}\right)$ and $\mathbf{v}_{i, k}^{*}=\left(0,0, w_{i, k}^{*}\right)$. In this equation, the material derivatives of $w_{l, j}^{*}$ and $w_{i, k}^{*}$ along paths of precipitation are ignored. The validation of this approximation is discussed in Ooyama [70]. For convenience, we abbreviate the advection term and Coriolis term to a and c, respectively:

$$
\mathbf{a} \equiv \nabla \cdot(\rho \mathbf{v} \otimes \mathbf{v}), \quad \mathbf{c} \equiv 2 \rho \mathbf{\Omega} \times \mathbf{v}
$$

The vertical velocity component and the horizontal velocity vector are $w=\mathbf{v} \cdot \hat{\mathbf{k}}$ and $\mathbf{v}_{h}=\mathbf{v}-w \hat{\mathbf{k}}$, respectively. From (B.7), the horizontal and the vertical momentum equations are given by

$$
\begin{aligned}
& \frac{\partial\left(\rho \mathbf{v}_{h}\right)}{\partial t}+\mathbf{a}_{h}+\mathbf{c}_{h}=-\nabla_{h} p+\mathbf{f}_{h} \\
& -\sum_{j=1}^{j_{\max }} \frac{1}{r^{2}} \frac{\partial\left(r^{2} \rho q_{l, j} w_{l, j}^{*} \mathbf{v}_{h}\right)}{\partial z}-\sum_{k=1}^{k_{\max }} \frac{1}{r^{2}} \frac{\partial\left(r^{2} \rho q_{i, k} w_{i, k}^{*} \mathbf{v}_{h}\right)}{\partial z} . \\
& \frac{\partial(\rho w)}{\partial t}+a_{z}+c_{z}=-\frac{\partial p}{\partial z}-\rho g+f_{z} \\
& -\sum_{j=1}^{j_{\max }} \frac{1}{r^{2}} \frac{\partial\left(r^{2} \rho q_{l, j} w_{l, j}^{*} w\right)}{\partial z}-\sum_{k=1}^{k_{\max }} \frac{1}{r^{2}} \frac{\partial\left(r^{2} \rho q_{i, k} w_{i, k}^{*} w\right)}{\partial z}
\end{aligned}
$$

$\underline{\text { Energy equation }}$

First, we summarize thermodynamic variables used in this model. Following Satoh [49], we neglect the dependence of specific heats on the temperature but consider the dependence of latent heats on temperature. The latent heats 
for vaporization and fusion are expressed respectively as

$$
L_{v}=L_{v 00}+\left(C_{p v}-C_{l}\right) T, \quad L_{f}=L_{f 00}+\left(C_{l}-C_{i}\right) T
$$

where $T$ is temperature; $C_{p v}$ is the specific heat at constant pressure for water vapor; $C_{l}$ and $C_{i}$ are the specific heats for liquid water and solid water; $L_{v 00}$ is the latent heat of vaporization at $0 \mathrm{~K}$ and $L_{f 00}$ is that of fusion at $0 \mathrm{~K}$. The enthalpy and internal energy per unit mass for each substances are defined as follows:

$$
\begin{aligned}
& h_{d}=C_{p d} T, \quad e_{d}=C_{v d} T, \\
& h_{v}=C_{p v} T+L_{v 00}, \quad e_{v}=C_{v v} T+L_{v 00}, \\
& h_{l, j}=C_{l} T, \quad e_{l, j}=h_{l, j}, \\
& h_{i, k}=C_{i} T-L_{f 00}, \quad e_{i, k}=h_{i, k},
\end{aligned}
$$

where $C_{p d}$ is the specific heat at constant pressure for dry air; $C_{v d}\left(=C_{p d}-R_{d}\right)$ and $C_{v v}\left(=C_{p v}-R_{v}\right)$ are the specific heats at the constant volume for dry air and water vapor, respectively, $R_{d}$ and $R_{v}$ are the gas constants for dry air and water vapor, respectively. The moist enthalpy per unit mass (including water substance), pressure, and the moist internal energy are respectively written as

$$
\begin{aligned}
h & =q_{d} C_{p d} T+q_{v}\left(C_{p v} T+L_{v 00}\right)+q_{l} C_{l} T+q_{i}\left(C_{i} T-L_{f 00}\right), \\
p & =\rho\left(q_{d} R_{d}+q_{v} R_{v}\right) T, \\
e & =h-\frac{p}{\rho} \\
& =q_{d} C_{v d} T+q_{v}\left(C_{v v} T+L_{v 00}\right)+q_{l} C_{l} T+q_{i}\left(C_{i} T-L_{f 00}\right) .
\end{aligned}
$$

It should be commented here that, in the forms of (B.10) and (B.12), enthalpy and internal energy of water substance are measured from those of liquid water at $0 \mathrm{~K}$. If enthalpy and internal energy of water substance are measured from those of solid water at $0 \mathrm{~K},(\mathrm{~B} .10)$ and (B.12) are replaced respectively by

$$
h=q_{d} C_{p d} T+q_{v}\left(C_{p v} T+L_{v 00}+L_{f 00}\right)+q_{l}\left(C_{l} T+L_{f 00}\right)+q_{i} C_{i} T
$$




$$
e=q_{d} C_{v d} T+q_{v}\left(C_{v v} T+L_{v 00}+L_{f 00}\right)+q_{l}\left(C_{l} T+L_{f 00}\right)+q_{i} C_{i} T
$$

The resulting conservation equation for the moist internal energy takes the form:

$$
\begin{aligned}
& \frac{\partial(\rho e)}{\partial t}+\nabla_{h} \cdot\left(\rho \mathbf{v}_{h} h\right)+\frac{1}{r^{2}} \frac{\partial\left(r^{2} \rho w h\right)}{\partial z}=\left(\mathbf{v}_{h} \cdot \nabla_{h} p+w \frac{\partial p}{\partial z}\right) \\
& -\left[\sum_{j=1}^{j_{\max }} \frac{1}{r^{2}} \frac{\partial\left(r^{2} \rho q_{l, j} e_{l, j} w_{l, j}^{*}\right)}{\partial z}+\sum_{k=1}^{k_{\max }} \frac{1}{r^{2}} \frac{\partial\left(r^{2} \rho q_{i, k} e_{i, k} w_{i, k}^{*}\right)}{\partial z}\right] \\
& -\left(\sum_{j=1}^{j_{\max }} \rho q_{l, j} g w_{l, j}^{*}+\sum_{k=1}^{k_{\max }} \rho q_{i, k} g w_{i, k}^{*}\right)-\left(\mathbf{v}_{h} \cdot \mathbf{f}_{h}+w f_{z}\right)+q_{h e a t},
\end{aligned}
$$

where $q_{\text {heat }}$ represents source term of internal energy, such as radiative forcing and convergence of latent and sensible heat fluxes. The first term on the right hand side represents the conversion from kinetic energy due to pressure force, the second term represents the vertical convergence of precipitating fluxes, and the third term represents the heat source by the drag force, which is converted from potential energy [70].

The moist internal energy (B.12) can be rewritten as

$$
e=C_{V} T+\left(q_{v} L_{v 00}-q_{i} L_{f 00}\right)=e_{a}+\left(q_{v} L_{v 00}-q_{i} L_{f 00}\right)
$$

where $e_{a} \equiv C_{V} T$ and $C_{V}=q_{d} C_{v d}+q_{v} C_{v v}+q_{l} C_{l}+q_{i} C_{i}$ represents the specific heat of total air at constant volume. From this, we regard the first term of (B.14) as the sensible heat part of internal energy, and the second term, $\left(q_{v} L_{v 00}-q_{i} L_{f 00}\right)$, as the latent heat part of internal energy. In the same way, we define the sensible heat part of enthalpy, which is denoted by $h_{a} \equiv h-\left(q_{v} L_{v 00}-q_{i} L_{f 00}\right)$. Multiplying the sum of (B.4) over $k$ by $L_{f 00}$ and (B.2) by $L_{v 00}$, and subtracting the two from (B.13), we obtain the equation of the sensible heat part of internal energy; that is 


$$
\begin{aligned}
& \frac{\partial\left(\rho e_{a}\right)}{\partial t}+\nabla_{h} \cdot\left(\rho \mathbf{v}_{h} h_{a}\right)+\frac{1}{r^{2}} \frac{\partial\left(r^{2} \rho w h_{a}\right)}{\partial z}=\left(\mathbf{v}_{h} \cdot \nabla_{h} p+w \frac{\partial p}{\partial z}\right) \\
& -\left[\sum_{j=1}^{j_{\max }} \frac{1}{r^{2}} \frac{\partial\left(r^{2} \rho q_{l, j} C_{l} T w_{l, j}^{*}\right)}{\partial z}+\sum_{k=1}^{k_{\max }} \frac{1}{r^{2}} \frac{\partial\left(r^{2} \rho q_{i, k} C_{i} T w_{i, k}^{*}\right)}{\partial z}\right] \\
& -\left(\sum_{j=1}^{j_{\max }} \rho q_{l, j} g w_{l, j}^{*}+\sum_{k=1}^{k_{\max }} \rho q_{i, k} g w_{i, k}^{*}\right) \\
& -\left(\mathbf{v}_{h} \cdot \mathbf{f}_{h}+w f_{z}\right)+q_{\text {heat }}-L_{v 00} s_{v}+L_{f 00} s_{i} .
\end{aligned}
$$

\section{The conservation of total energy}

We demand that the total energy is conserved in our model. In the following, we review some pertinent points. The kinetic energy is defined by

$$
k=\frac{\rho\left(\mathbf{v}_{h} \cdot \mathbf{v}_{h}+w^{2}\right)}{2} .
$$

The equation of kinetic energy can be derived from the momentum equations:

$$
\begin{aligned}
& \frac{\partial(\rho k)}{\partial t}+\nabla_{h} \cdot\left(\rho \mathbf{v}_{h} k\right)+\frac{1}{r^{2}} \frac{\partial\left(r^{2} \rho w k\right)}{\partial z}=-\left(\mathbf{v}_{h} \cdot \nabla_{h} p+w \frac{\partial p}{\partial z}\right)-\rho g w \\
& -\left[\sum_{j=1}^{j_{\max }} \frac{1}{r^{2}} \frac{\partial\left(r^{2} \rho q_{l, j} w_{l, j}^{*} k\right)}{\partial z}+\sum_{k=1}^{k_{\max }} \frac{1}{r^{2}} \frac{\partial\left(r^{2} \rho q_{i, k} w_{i, k}^{*} k\right)}{\partial z}\right] \\
& +\mathbf{v}_{h} \cdot \mathbf{f}_{h}+w f_{z} .
\end{aligned}
$$

The equation of potential energy is simply $\rho d \phi / d t=\rho g w$, where geopotential $\phi=g z$. The flux-form equation of potential energy is derived from this equation, using the total-mass continuity (B.6); that is,

$$
\begin{aligned}
& \frac{\partial(\rho \phi)}{\partial t}+\nabla_{h} \cdot\left(\rho \mathbf{v}_{h} \phi\right)+\frac{1}{r^{2}} \frac{\partial\left(r^{2} \rho w \phi\right)}{\partial z} \\
= & \rho g w+\sum_{j=1}^{j_{\max }} \rho q_{l, j} g w_{l, j}^{*}+\sum_{k=1}^{k_{\max }} \rho q_{i, k} g w_{i, k}^{*} \\
& -\left[\sum_{j=1}^{j_{\max }} \frac{1}{r^{2}} \frac{\partial\left(r^{2} \rho q_{l, j} w_{l, j}^{*} \phi\right)}{\partial z}+\sum_{k=1}^{k_{\max }} \frac{1}{r^{2}} \frac{\partial\left(r^{2} \rho q_{i, k} w_{i, k}^{*} \phi\right)}{\partial z}\right] .
\end{aligned}
$$


The sum of (B.13), (B.17), and (B.16) gives the conservation law of the total energy. If we use the sensible heat part of internal energy $e_{a}$ instead of $e$, the equation of the corresponding total energy can be written as

$$
\begin{aligned}
& \frac{\partial\left[\rho\left(e_{a}+k+\phi\right)\right]}{\partial t}+\nabla_{h}\left[\rho \mathbf{v}_{h}\left(h_{a}+k+\phi\right)\right]+\frac{1}{r^{2}} \frac{\partial\left[r^{2} \rho w\left(h_{a}+k+\phi\right)\right]}{\partial z} \\
= & -\left\{\sum_{j=1}^{j_{\max }} \frac{1}{r^{2}} \frac{\partial\left[r^{2} \rho q_{l, j}\left(C_{l} T+k+\phi\right) w_{l, j}^{*}\right]}{\partial z}\right. \\
& \left.+\sum_{k=1}^{k_{\max }} \frac{1}{r^{2}} \frac{\partial\left[r^{2} \rho q_{i, k}\left(C_{i} T+k+\phi\right) w_{i, k}^{*}\right]}{\partial z}\right\} \\
& +q_{\text {heat }}-L_{v 00} s_{v}+L_{f 00} s_{i} .
\end{aligned}
$$

$\underline{\text { Summary of the governing equations }}$

Here, we summarize the governing equations of the model: the continuity equations are (B.6) for total mass, (B.2) for water vapor, and (B.3) and (B.4) for each component of liquid and solid water substances. The momentum equations are (B.8) for the horizontal components and (B.9) for the vertical component of momentum. As for energy, we use the sensible heat part of internal energy equation (B.15). Additionally, we use the conservation of total energy (B.18). Equations are discretized in the flux form by defining control volumes in space. Thus, the prognostic variables are $\left(\rho, \rho \mathbf{v}_{h}, \rho w, \rho e_{a}, \rho q_{v}, \rho q_{l, j}, \rho q_{i, j}\right)$. The other variables, such as $p$ and $T$, are derived diagnostically from these prognostic variables.

\section{References}

[1] W. Ohfuchi, H. Nakamura, M.K. Yoshioka, T. Enomoto, K. Takaya, X. Peng, S. Yamane, T. Nishimura, Y. Kurihara, and K. Ninomiya, 10-km 
mesh meso-scale resolving simulations of the global atmosphere on the Earth Simulator - Preliminary outcomes of AFES (AGCM for the Earth Simulator), J. the Earth Simulator, 1, 8 (2004).

[2] R. Mizuta, K. Oouchi, H. Yoshimura, A. Noda, K. Katayama, S. Yukimoto, M. Hosaka, S. Kusunoki, H. Kawai, and M. Nakagawa, 20km-mesh global climate simulations using JMA-GSM model -mean climate states-, J. Meteor. Soc. Japan, 84, 165 (2005).

[3] M. Hortal, TL2047 at European Centre for Medium-Range Weather Forecasts, presentation at the 2006 Workshop on solutions of partial differential equations on the sphere, Monterey, 2006.

[4] A. Arakawa, The cumulus parameterization problem: Past, present, and future, J. Clim., 17, 2493 (2004).

[5] G. R. Stuhne and W. R. Peltier, Vortex erosion and amalgamation in a new model of large scale flow on the sphere, J. Comput. Phys., 128, 58 (1996).

[6] M. Taylor, J. Tribbia, and M. Iskandarani, The spectral element method for the shallow water equations on the sphere, J. Comput. Phys., 130, 92 (1997).

[7] D. A. Randall, R. Heikes, and T. Ringler, Global atmospheric modeling using a geodesic grid with an isentropic vertical coordinate. Chapter 17 of "General Circulation Model Development", Academic Press, 509 (2000).

[8] M. Satoh, H. Tomita, H. Miura, S. Iga, and T. Nasuno, Development of a global cloud resolving model - a multi-scale structure of tropical convections -, J. Earth Simulator, 3, 11 (2005).

[9] H.-B. Cheong, A Dynamical Core with Double Fourier Series: Comparison with the Spherical Harmonics Method, Mon. Wea. Rev., 134, 1299 (2006). 
[10] H.-B. Cheong, Double Fourier series on a sphere: applications to elliptic and vorticity equations, J. Comput. Phys., 157, 327 (2000).

[11] H.-B. Cheong, Application of double Fourier series to the shallow-water equations on a sphere, J. Comput. Phys., 165, 261 (2000).

[12] I.-H. Kwon, H.-B. Cheong, M. Joh, I.-U. Chung, C.-H. Cho, and W.J. Lee, Application of double-Fourier-series spectral method to a huge size problem: two-dimensional simulations of the shear instability on the sphere, J. Met. Soc. Japan, 82, 1301 (2004).

[13] F. H. M. Semazzi, J. H. Qian, and J. S. Scroggs, A global nonhydrostatic semi-Lagrangian atmospheric model without orography, Mon. Wea. Rev., 123, 2534 (1995).

[14] M. J. P. Cullen, T. Davies, M. H. Mawson, J. A. James, S. C. Coutler, and, A. Malcolm, An overview of numerical methods for the next generation U.K. NWP and climate model, Numerical methods in Atmospheric and Ocean Modelling, The Andrew J. Robert Memorial Volume, edited by C. A. Lin (NRC Research Press, 1997), p.425.

[15] J. H. Qian, F. H. M. Semazzi, and J. S. Scroggs, A global nonhydrostatic semi-Lagrangian atmospheric model with orography, Mon. Wea. Rev., 126, 747 (1998).

[16] J. Côté, S. Granvel, A. Méthot, and A. Patoine, The operational CMCMRB global environmental multiscale (GEM) model. Part I : Design considerations and formulation, Mon. Wea. Rev., 126, 1373 (1998).

[17] J. Côté, G. Desmarais, S. Granvel, A. Méthot, A. Patoine, M. Roch, and A. Staniforth, The operational CMC-MRB global environmental multiscale (GEM) model. Part II : Results, Mon. Wea. Rev., 126, 1397 (1998).

[18] K.-S. Yeh, J. Côté, S. Gravel, A. Methot, A. Patoine, M. Roch, A. Staniforth, The CMC-MRB global environmental multiscale (GEM) model. 
Part III: Nonhydrostatic formulation, Mon. Wea. Rev., 120, 329-356 (2002).

[19] T. Davies, M. J. P. Cullen, A. J. Malcolm, M. H. Mawson, A. Staniforth, A. A. White, N. Wood, A new dynamical core for the Met Office's global and regional modelling of the atmosphere, Quart. J. Roy. Meteor. Soc., 131, 1759-1782 (2005).

[20] J. L. McGregor, Semi-Lagrangian advection on conformal-cubic grids, Mon. Wea. Rev., 124, 1311 (1996).

[21] M. Rancic, R. J. Purser, F. Mesinger, A global shallow-water model using an expanded spherical cube: Gnomonic versus conformal coordinates, $Q$. J. R. Met. Soc., 122, 959 (1996).

[22] R. J. Purser and M. Rancic, Smooth quasi-homogeneous gridding of the sphere, Q. J. R. Meteor. Soc., 124, 637 (1998).

[23] A. Adcroft, J.-M. Campin, C. Hill, and J. Marshall, Implementation of an atmosphere-ocean general circulation model on the expanded spherical cube, Mon. Wea. Rev., 132, 2845 (2004).

[24] J. Dudhia and J. F. Bresch, A global version of PSU-NCAR Mesoscale Model, Mon. Wea. Rev., 130, 2989 (2002).

[25] R. Swinbank and R. J. Purser, Fibonacci grid: A novel approach to global modelling, Q. J. Roy. Met. Soc., 132, 1769-1793 (2006).

[26] J. Dudhia, A nonhydrostatic version of the Penn State-NCAR mesoscale model: Validation tests and simulation of an Atlantic cyclone and cold front, Mon. Wea. Rev., 121, 1493 (1993).

[27] J. B. Klemp and R. B. Wilhemson, The simulation of three-dimensional convective storm dynamics, J. Atmos. Sci., 35, 1070 (1978).

[28] D. L. Williamson, Integration of the barotropic vorticity equation on a spherical geodesic grid, Tellus, 20, 642 (1968). 
[29] R. Sadourny, A. Arakawa, and Y. Mintz, Integration of the nondivergent barotropic vorticity equation with an icosahedral hexagonal grid for the sphere, Mon. Wea. Rev., 96, 351 (1968).

[30] R. Sadourny, Numerical integration of the primitive equations on a spherical grid with hexagonal cells, in Proceedings of the WMO/IUGG Symposium on Numerical Weather Prediction in Tokyo, Tech. Rep. of JMA (Japan Meteorological Agency, Nov. 26-Dec. 4., 1969), pp. VII45-VII52.

[31] D. L. Williamson, Integration of the primitive barotropic model over a spherical geodesic grid, Mon. Wea. Rev., 98, 512 (1969).

[32] M. J. P. Cullen, Integration of the primitive equations on a sphere using the finite element method, Quart. J. R. Met. Soc., 100, 555 (1974).

[33] M. J. P. Cullen and C. D. Hall, Forecasting and general circulation results from finite element models, Quart. J. R. Met. Soc., 100, 571 (1979).

[34] Y. Masuda and H. Ohnishi, An integration scheme of the primitive equation model with an icosahedral-hexagonal grid system and its application to the shallow water equations. in Short- and Medium-Range Numerical Weather Prediction. Collection of Papers Presented at the WMO/IUGG NWP Symposium, Tokyo, Aug.4-8, 1986 (Japan Meteorological Society, 1986), p.317.

[35] R. H. Heikes and D. A. Randall, Numerical integration of the shallowwater equations on a twisted icosahedral grid, Part I: Basic design and results of tests, Mon. Wea. Rev., 123, 1862 (1995).

[36] R. H. Heikes and D. A. Randall, Numerical integration of the shallowwater equations on a twisted icosahedral grid. Part II: A detailed description of the grid and analysis of numerical accuracy, Mon. Wea. Rev., 123, 1881 (1995).

[37] T. D. Ringler, R. H. Heikes, and D. A. Randall, Modeling the atmo- 
spheric general circulation using a spherical geodesic grid: A new class of dynamical cores, Mon. Wea. Rev., 128, 2471 (2000).

[38] J. Steppeler and P. Prohl, Application of finite volume methods to atmospheric models, Beitr. Phys. Atmosph., 69, 297 (1996).

[39] D. Majewski, D. Liermann, P. Prohl, B. Ritter, M. Buchhold, T. Hanisch, G. Paul, and W. Wergen, The operational global icosahedral-hexagonal gridpoint model GME: Description and high-resolution tests, Mon. Wea. Rev., 130, 319 (2002).

[40] L. Bonaventura, The ICON project: Development of a unified model using triangular geodesic grid, in Proceedings of the ECMWF Annual Seminar on Developments in Numerical Methods for Atmospheric and Ocean Modelling ECMWF, Reading, 2004.

[41] G. R. Stuhne and W. R. Peltier, New icosahedral grid-point discretizations of the shallow water equations on the sphere, J. Comput. Phys., 148, 23 (1999).

[42] J. Thuburn, A PV-based shallow-water model on a hexagonal-icosahedral grid, Mon. Wea. Rev., 125, 2328 (1997).

[43] H. Tomita, M. Tsugawa, M. Satoh, and K. Goto, Shallow water model on a modified icosahedral geodesic grid by using spring dynamics, J. Comp. Phys., 174, 579-613 (2001).

[44] H. Tomita, M. Satoh, and K. Goto, An optimization of icosahedral grid by using spring dynamics, J. Comp. Phys., 183, 307 (2002).

[45] H. Miura and M. Kimoto, A comparison of grid quality of optimized spherical hexagonal-pentagonal geodesic grids, Mon. Wea. Rev., 133, 2817 (2005).

[46] The Earth Similator Center. http://www.es.jamstec.go.jp/esc/eng/.

[47] P. K. Smolarkiewicz, L. G. Margolin, A. Wyszogrodzki, A class of non- 
hydrostatic global models, J. Atmos. Sci., 58, 349 (2001).

[48] M. Satoh, Conservative scheme for the compressible non-hydrostatic models with the horizontally explicit and vertically implicit time integration scheme, Mon. Wea. Rev., 130, 1227 (2002).

[49] M. Satoh, Conservative scheme for a compressible nonhydrostatic model with moist processes, Mon. Wea. Rev., 131, 1033 (2003).

[50] H. Tomita and M. Satoh, A new dynamical framework of nonhydrostatic global model using the icosahedral grid, Fluid Dyn. Res., 34, 357 (2004).

[51] S. Iga, H. Tomita, M. Satoh, and K. Goto, Mountain-wave-like spurious waves due to inconsistency of horizontal and vertical resolution associated with cold fronts, Mon. Wea. Rev. (2007), accepted.

[52] H. Tomita, H. Miura, S. Iga, T. Nasuno, and M. Satoh, A global cloudresolving simulation: preliminary results from an aqua planet experiment, Geophys. Res. Lett., 32, L08805 (2005), doi:10.1029/2005GL022459.

[53] H. Miura, H. Tomita, T. Nasuno, S. Iga, M. Satoh, and T. Matsuno, A climate sensitivity test using a global cloud resolving model under an aqua planet condition, Geophys. Res. Lett., 32, L19717 (2005), doi:10.1029/2005GL023672.

[54] T. Nasuno, H. Tomita, S. Iga, H. Miura, and M. Satoh, Multi-scale organization of convection simulated with explicit cloud processes on an aqua planet, J. Atmos. Sci. (2007), accepted.

[55] R. B. Neale and B. J. Hoskins, A standard test for AGCMs including their physical parameterizations: I: The proposal, Atmospheric Science Letter, 1 (2001), doi:10.1006/asle.2000.0019.

[56] A. Arakawa and S. Moorthi, Baroclinic instability in vertically discrete system, J. Atmos. Sci., 45, 1688 (1988).

[57] M. S. Fox-Rabinovitz, Computational dispersion properties of 3D stag- 
gered grids for a nonhydrostatic anelastic system, Mon. Wea. Wev., 124, 498 (1996).

[58] J. Thuburn, N. Wood, and A. Staniforth, Normal modes of deep atmospheres. I: Spherical geometry, Q. J. Roy. Met. Soc., 128, 1771 (2002).

[59] J. Thuburn and T. J. Woollings, Vertical discretizations for compressible Euler equation atmospheric models giving optimal representation of normal modes, J. Comp. Phys., 203, 386 (2005).

[60] K. Saito, G. Doms, U. Schaettler, and J. Steppeler, 3-D Mountain Waves by the Local-Modell of DWD and the MRI Mesoscale Nonhydrostatic Model, Papers in Meteorology and Geophysics, 49, 7 (1998).

[61] K. Saito, T. Fujita, Y. Yamada, J. Ishida, K. Kumagai, K. Aranami, S. Ohmori, R. Nagasawa, S. Kumagai, C. Muroi, T. Kato, H. Eito, and Y. Yamazaki, The Operational JMA Nonhydrostatic Mesoscale Model, Mon. Wea. Rev., 134, 1266 (2006).

[62] L. J. Wicker and W. C. Skamarock, Time-splitting scheme for the elastic equations incorporating second-order runge-Kutta time differencing, Mon. Wea. Rev., 126, 1992 (1998).

[63] L. J. Wicker and W. C. Skamarock, Time-Splitting Methods for Elastic Models Using Forward Time Schemes, Mon. Wea. Rev., 130, 2088 (2002).

[64] D. Mesinger, Numerical methods: The Arakawa approach, horizontal grid, global, and limited-area modeling, in "General Circulation Model Development", edited by D. A. Randall (Academic Press, 2000) p. 373.

[65] D. A. Randall, Geostrophic adjustment and finite-difference shallow water equations, Mon. Wea. Rev., 122, 1371 (1994).

[66] T. D. Ringler and D. A. Randall, A Potential Enstrophy and Energy Conserving Numerical Scheme for Solution of the Shallow-Water Equations on a Geodesic Grid, Mon. Wea. Rev., 130, 1397 (2002). 
[67] T. D. Ringler and D. A. Randall, The ZM Grid: An Alternative to the Z Grid, Mon. Wea. Rev., 130, 1411 (2002).

[68] D. L. Williamson, J. B. Drake, J. J. Hack, R. Jakob, and P. N. Swarztrauber, A standard test set for numerical approximations to the shallow water equations in spherical geometry, J. Comp. Phys., 102, 211 (1992).

[69] F. Xiao, T. Okazaki, and M. Satoh, An accurate semi-Lagrangian scheme for rain drop sedimentation, Mon. Wea. Rev., 131, 974 (2003).

[70] K. V. Ooyama, A dynamic and thermodynamic foundation for modeling the moist atmosphere with parameterized microphysics, J. Atmos. Sci, 58, $2073(2001)$.

[71] W. C. Skamarock and J. B. Klemp, The stability of time-split numerical methods for the hydrostatic and the nonhydrostatic elastic equations, Mon. Wea. Rev., 120, 2109 (1992).

[72] P. Jöckel, R. von Kuhlmann, M. G. Lawrence, B. Steil, C. A. M. Brenninkmeijer, P. J. Crutzen, P. J. Rasch, and B. Eaton, On a fundamental problem in implementing flux-form advection schemes for tracer transport in 3-dimensional general circulation and chemistry transport models, $Q$. J. Roy. Meteor. Soc., 127, 1035 (2001).

[73] E. S. Gross, L. Bonaventura, and G. Rosatti, Consistency with continuity in conservative advection schemes for free-surface models, Int. J. Numer. Meth. Fluids, 38, 307 (2002).

[74] W. W. Grabowski, Toward cloud resolving modeling of large-scale tropical circulations: A simple cloud microphysics parameterization, J. Atmos. Sci., 55, 3283 (1998).

[75] T. Nakazawa, Tropical super clusters within intraseasonal variations over the western Pacific, J. Meteor. Soc. Japan, 66, 823 (1988).

[76] Y. N. Takayabu, T. Iguchi, M. Kachi, A. Shibata, and H. Kanzawa, 
Abrupt termination of the 1997-98 El Nino in response to a MaddenJulian oscillation, Nature, 402, 279 (1999).

[77] Intercomparison diagnostics, The Aqua-Planet Experiment workshop, 22-25 Apr. 2005, Reading. http://www.met.reading.ac.jp//mike/APE/workshop2005.html.

[78] H. Miura, M. Satoh, H. Tomita, A. Noda, T. Nasuno, and S. Iga, A shortduration global cloud-resolving simulation under a realistic land and sea distribution. Geophys. Res. Lett. (2007), accepted.

[79] R. N. B. Smith, A scheme for predicting layer clouds and their water content in a general circulation model, Q. J. Roy. Soc. Met., 116, 435 (1990).

[80] N. A. Phillips, The general circulation of the atmosphere: a numerical experiment, Quart. J. Roy. Met. Soc., 82, 123 (1956).

[81] K. Nakajima and T. Matsuno, Numerical experiments concerning the origin of cloud clusters in the tropical atmosphere, J. Meteor. Soc. Japan, 66, 309 (1988).

[82] T. Matsuno, Toward the realization of cloud cluster-resolving global atmosphere models. Lecture presented at the 22nd General Assembly of IUGG at Birmingham, 18-30 July 1999, Session MW03 "Development of high resolution climate models" (1999).

[83] E. J. Zipser, Mesoscale and convective-scale downdrafts as distinct components of squall-line structure, Mon. Wea. Rev., 105, 1568 (1977).

[84] R. A. Houze,Jr., S. G. Geotis, F. D. Marks,Jr., and A. K. West,, Winter monsoon convection in the vicinity of north borneo. Part I: structure and time variation of the clouds and precipitation, Mon. Wea. Rev., 109, 1595 (1981).

[85] S. S. Chen and R.A. Houze Jr., Diurnal variation and life-cycle of deep 
convective systems over the tropical Pacific warm pool, Quart. J. Roy. Meteor. Soc., 123, 357 (1997).

[86] Y.-Y. Hayashi and A. Sumi, The 30-40 day oscillations simulated in an aqua-planet model, J. Meteorol. Soc. Japan, 64, 451 (1986).

[87] Y. N. Takayabu, Large-scale cloud disturbances associated with equatorial waves. Part I: Spectral features of the cloud disturbances, J. Meteor. Soc.Japan, 72, 433 (1994).

[88] M. Wheeler and G. N. Kiladis, Convectively coupled equatorial waves: analysis of clouds and temperature in the wavenumber-frequency domain, J. Atmos. Sci., 56, 374 (1999).

[89] M. Wheeler, G. N. Kiladis, and P. J. Webster, Large-scale dynamical fields associated with convectively coupled equatorial waves, J. Atmos. Sci., 57, 613 (2000).

[90] R. A. Madden and P. R. Julian, Description of a 40-50 day oscillation in the Tropics, J. Atmos. Sci., 28, 702 (1971).

[91] R. A. Madden and P. R. Julian, Description of global-scale circulation cells in the tropics with a 40-50 day period, J. Atmos. Sci., 29, 1109 $(1972)$

[92] J. M. Slingo, P. M. Innes, and K. R. Sperber, Modeling, in "Intraseasonal Variability in the Atmophere- Ocean Climate System”, Ed. W. K. M. Lau and D. E. Waliser (Springer, 2005), p.361.

[93] C.-P. Chang, Westward propagating cloud patterns in the tropical Pacific as seen from time-composite satellite photographs, J. Atmos. Sci., 27, 133 (1971)

[94] J. G. Charney and A. Eliassen, On the growth of the hurricane depression, J. Atmos. Sci., 21, 68 (1964).

[95] M. Satoh, T. Nasuno, H. Miura, H. Tomita, S. Iga, and Y. Takayabu, 
Precipitation statistics comparison between global cloud resolving simulation with NICAM and TRMM PR data. "High Resolution Numerical Modelling of the Atmosphere and Ocean", edited by Wataru Ohfuchi and Kevin Hamilton, (Springer, 2007), accepted.

[96] T. Gal-Chen and C.J. Somerville, On the use of a coordinate transformation for the solution of the Navier-Stokes equations, J. Comput. Phys., bf 17209 (1975).

[97] N.P. Wedi and P.K. Smolarkiewicz, Extending Gal-Chen and Somerville terrain-following coordinate transformation on time dependent curvilinear boundaries, J. Comput. Phys., 1931 (2004).

[98] Y.-L. Lin, R. D. Farley, H. D. Orville, Bulk parameterization of the snow field in a cloud model, J. Climate Applied Meteorology, 22, 1065 (1983).

[99] E. Kessler, On the distribution and continuity of water substance in atmospheric circulations, Meteorologial Monograph, 32 (American Meteorogical Society, 1969), p.1.

[100] W. W. Grabowski, A parameterization of cloud microphysics for longterm cloud-resolving modeling of tropical convection, Atmos. Res., 52, 17 (1999). 


\begin{tabular}{|c|c|c|}
\hline symbol & description & unit \\
\hline$\lambda, \theta$ & longitude, latitude & \\
\hline$r$ & distance from the center of the earth & {$[\mathrm{m}]$} \\
\hline$r_{0}$ & radius at the mean sea level (the earth radius) & {$[\mathrm{m}]$} \\
\hline$\gamma \equiv r / r_{0}$ & normalized radial distance & \\
\hline$z=r-r_{0}$ & height from the mean sea level & {$[\mathrm{m}]$} \\
\hline$t$ & time & {$[\mathrm{s}]$} \\
\hline$\hat{\mathbf{i}}, \hat{\mathbf{j}}, \hat{\mathbf{k}}$ & $\begin{array}{l}\text { unit vectors in the longitudinal, latitudinal, and } \\
\text { outward directions }\end{array}$ & \\
\hline$\varphi$ & scalar quantity & \\
\hline $\mathbf{u}$ & vector quantity & \\
\hline$\left(u_{\lambda}, u_{\theta}, u_{z}\right)=\mathbf{u} \cdot(\hat{\mathbf{i}}, \hat{\mathbf{j}}, \hat{\mathbf{k}})$ & components of $\mathbf{u}$ & \\
\hline $\mathbf{u}_{h}=\mathbf{u}-\hat{\mathbf{k}} u_{z}$ & horizontal vector of $\mathbf{u}$ & \\
\hline$\nabla, \nabla \cdot$ & three-dimensional gradient operator, divergence operator & {$\left[\mathrm{m}^{-1}\right]$} \\
\hline$\nabla_{h}, \nabla_{h}$ & $\begin{array}{l}\text { two-dimensional gradient operator, spherical di- } \\
\text { vergence operator }\end{array}$ & {$\left[\mathrm{m}^{-1}\right]$} \\
\hline$\nabla_{h 0}=\gamma \nabla_{h}$ & normalized two-dimensional gradient operator & {$\left[\mathrm{m}^{-1}\right]$} \\
\hline
\end{tabular}

Table 1(1): Description of symbols in the text. 


\begin{tabular}{|c|c|c|}
\hline symbol & description & unit \\
\hline$\xi=\xi(z)$ & an arbitrary monotonic function of height & \\
\hline$z_{T}$ & the top of the model domain & {$[\mathrm{m}]$} \\
\hline$z_{s}$ & the surface height & {$[\mathrm{m}]$} \\
\hline$\tilde{\nabla}_{h 0}$ & two-dimensional gradient operator on a constant $\xi$ shell & \\
\hline$G^{1 / 2} \equiv\left(\frac{\partial z}{\partial \xi}\right)_{h}$ & metric & \\
\hline $\mathbf{G}^{\mathbf{z}} \equiv \nabla_{h 0} \xi$ & metric & \\
\hline$\rho$ & total density of moist air & {$\left[\mathrm{kg} \mathrm{m}^{-3}\right]$} \\
\hline$q_{v}$ & mass concentration of vapor & {$\left[\mathrm{kg} \mathrm{kg}^{-1}\right]$} \\
\hline$l_{\max }, k_{\max }$ & total numbers of liquid/solid water components & \\
\hline$q_{l, j}\left(j=1, \cdots, l_{\max }\right)$ & mass concentration of $j$-th component of liquid water & {$\left[\mathrm{kg} \mathrm{kg}^{-1}\right]$} \\
\hline$q_{i, k}\left(k=1, \cdots, k_{\max }\right)$ & mass concentration of $k$-th component of solid water & {$\left[\mathrm{kg} \mathrm{kg}^{-1}\right]$} \\
\hline$q_{l}=\sum_{j=1}^{l_{\max }} q_{l, j}$ & mass concentration of liquid phase of water & {$\left[\mathrm{kg} \mathrm{kg}^{-1}\right]$} \\
\hline$q_{i}=\sum_{k=1}^{k_{\max }} q_{i, k}$ & mass concentration of solid phase of water & {$\left[\mathrm{kg} \mathrm{kg}^{-1}\right]$} \\
\hline$q_{w}=q_{v}+q_{l}+q_{i}$ & mass concentration of total water & {$\left[\mathrm{kg} \mathrm{kg}^{-1}\right]$} \\
\hline$q_{d}=1-q_{w}$ & mass concentration of dry air & {$\left[\mathrm{kg} \mathrm{kg}^{-1}\right]$} \\
\hline$s_{n}$ & mass source term of $n$-th component & {$\left[\mathrm{kg} \mathrm{m}^{-3} \mathrm{~s}^{-1}\right]$} \\
\hline
\end{tabular}

Table 1(2): Description of symbols in the text (cont.). 


\begin{tabular}{|c|c|c|}
\hline symbol & description & unit \\
\hline $\mathbf{v}$ & velocity vector of the air & {$\left[\mathrm{m} \mathrm{s}^{-1}\right]$} \\
\hline$w=\mathbf{v} \cdot \hat{\mathbf{k}}$ & vertical velocity of the air & {$\left[\mathrm{m} \mathrm{s}^{-1}\right]$} \\
\hline $\mathbf{v}_{h}=\mathbf{v}-w \hat{\mathbf{k}}$ & horizontal velocity vector of the air & {$\left[\mathrm{m} \mathrm{s}^{-1}\right]$} \\
\hline $\begin{array}{l}w_{l, j}^{*}, w_{i, k}^{*}(j=1, \cdots, \\
\left.l_{\max }, k=1, \cdots, k_{\max }\right)\end{array}$ & terminal velocities relative to the air & {$\left[\mathrm{m} \mathrm{s}^{-1}\right]$} \\
\hline $\mathbf{v}_{l, j}^{*}=\left(0,0, w_{l, j}^{*}\right), \mathbf{v}_{i, k}^{*}=\left(0,0, w_{i, k}^{*}\right)$ & terminal velocity vectors relative to the air & {$\left[\mathrm{m} \mathrm{s}^{-1}\right]$} \\
\hline$p$ & pressure & {$[\mathrm{Pa}]$} \\
\hline$T$ & temperature & {$[\mathrm{K}]$} \\
\hline$g$ & acceleration due to gravity & {$\left[\mathrm{m} \mathrm{s}^{-2}\right]$} \\
\hline$\Omega$ & angular velocity of the earth & {$\left[\mathrm{rad} \mathrm{s}^{-2}\right]$} \\
\hline f & frictional force & {$\left[\mathrm{kg} \mathrm{m}^{-2} \mathrm{~s}^{-2}\right]$} \\
\hline $\mathbf{a}=\nabla \cdot(\rho \mathbf{v} \otimes \mathbf{v})$ & advection term of the momentum equation & {$\left[\mathrm{kg} \mathrm{m}^{-2} \mathrm{~s}^{-2}\right]$} \\
\hline $\mathbf{c}=2 \rho \boldsymbol{\Omega} \times \mathbf{v}$ & Coriolis force term & {$\left[\mathrm{kg} \mathrm{m}^{-2} \mathrm{~s}^{-2}\right]$} \\
\hline
\end{tabular}

Table 1(3): Description of symbols in the text (cont.). 


\begin{tabular}{|c|c|c|}
\hline symbol & description & unit \\
\hline$L_{v}, L_{f}$ & latent heats for vaporization and fusion & {$\left[\mathrm{J} \mathrm{kg}^{-1}\right]$} \\
\hline$L_{v 00}, L_{f 00}$ & latent heats for vaporization and fusion at $0 \mathrm{~K}$ & {$\left[\mathrm{~J} \mathrm{~kg}^{-1}\right]$} \\
\hline$C_{p d}$ & the specific heat at constant pressure for dry air & {$\left[\mathrm{J} \mathrm{kg}^{-1} \mathrm{~K}^{-1}\right]$} \\
\hline$C_{p v}$ & the specific heat at constant pressure for water vapor & {$\left[\mathrm{J} \mathrm{kg}^{-1} \mathrm{~K}^{-1}\right]$} \\
\hline$C_{l}, C_{i}$ & the specific heat for liquid/solid water & {$\left[\mathrm{J} \mathrm{kg}^{-1} \mathrm{~K}^{-1}\right]$} \\
\hline$h_{n}$ & enthalpy of $n$-th component per unit mass & {$\left[\mathrm{J} \mathrm{kg}^{-1}\right]$} \\
\hline$e_{n}$ & internal energy of $n$-th component per unit mass & {$\left[\mathrm{J} \mathrm{kg}^{-1}\right]$} \\
\hline$R_{d}$ & gas constant for dry air & {$\left[\mathrm{J} \mathrm{kg}^{-1} \mathrm{~K}^{-1}\right]$} \\
\hline$R_{v}$ & gas constant for water vapor & {$\left[\mathrm{J} \mathrm{kg}^{-1} \mathrm{~K}^{-1}\right]$} \\
\hline$h$ & moist enthalpy per unit mass & {$\left[\mathrm{J} \mathrm{kg}^{-1}\right]$} \\
\hline$e$ & moist internal energy per unit mass & {$\left[\mathrm{J} \mathrm{kg}^{-1}\right]$} \\
\hline$q_{\text {heat }}$ & source term of internal energy & {$\left[\mathrm{W} \mathrm{m} \mathrm{m}^{-3}\right]$} \\
\hline $\begin{array}{l}C_{V}=q_{d} C_{v d}+q_{v} C_{v v}+ \\
q_{l} C_{l}+q_{i} C_{i}\end{array}$ & specific heat of total air at constant volume & {$\left[\mathrm{J} \mathrm{kg}^{-1} \mathrm{~K}^{-1}\right]$} \\
\hline$e_{a} \equiv C_{V} T$ & sensible heat part of internal energy & {$\left[\mathrm{J} \mathrm{kg}^{-1}\right]$} \\
\hline$h_{a} \equiv h-\left(q_{v} L_{v 00}-q_{i} L_{f 00}\right)$ & sensible heat part of enthalpy & {$\left[\mathrm{J} \mathrm{kg}^{-1}\right]$} \\
\hline$k$ & kinetic energy & {$\left[\mathrm{m}^{2} \mathrm{~s}^{-2}\right]$} \\
\hline$\phi$ & geopotential & {$\left[\mathrm{m}^{2} \mathrm{~s}^{-2}\right]$} \\
\hline
\end{tabular}

Table 1(4): Description of symbols in the text (cont.). 


\begin{tabular}{|c|c|c|}
\hline symbol & description & unit \\
\hline$\rho_{s}$ & density of a basic state & {$\left[\mathrm{kg} \mathrm{m}^{-3}\right]$} \\
\hline$p_{s}$ & pressure of a basic state & {$[\mathrm{Pa}]$} \\
\hline$\rho^{\prime}=\rho-\rho_{s}$ & density perturbation & {$\left[\mathrm{kg} \mathrm{m}^{-3}\right]$} \\
\hline$p^{\prime}=p-p_{s}$ & pressure perturbation & {$[\mathrm{Pa}]$} \\
\hline$R=G^{1 / 2} \gamma^{2} \rho^{\prime}$ & prognostic variable of density & {$\left[\mathrm{kg} \mathrm{m}^{-3}\right]^{\dagger}$} \\
\hline$P=G^{1 / 2} \gamma^{2} p^{\prime}$ & pressure perturbation & {$[\mathrm{Pa}]^{\dagger}$} \\
\hline $\mathbf{V}_{h}=G^{1 / 2} \gamma^{2} \rho \mathbf{v}_{h}$ & $\begin{array}{l}\text { prognostic variable of horizontal momentum } \\
\text { vector }\end{array}$ & {$\left[\mathrm{kg} \mathrm{m}^{-2} \mathrm{~s}^{-1}\right]^{\dagger}$} \\
\hline$W=G^{1 / 2} \gamma^{2} \rho w$ & prognostic variable of vertical momentum & {$\left[\mathrm{kg} \mathrm{m}^{-2} \mathrm{~s}^{-1}\right]^{\dagger}$} \\
\hline$E_{a}=G^{1 / 2} \gamma^{2} \rho e_{a}$ & $\begin{array}{l}\text { prognostic variable of sensible heat part of in- } \\
\text { ternal energy }\end{array}$ & {$\left[\mathrm{J} \mathrm{m}^{-3}\right]^{\dagger}$} \\
\hline$E_{t o t}=G^{1 / 2} \gamma^{2} \rho e_{t o t}$ & total energy & {$\left[\mathrm{J} \mathrm{m}^{-3}\right]^{\dagger}$} \\
\hline$Q_{n}=G^{1 / 2} \gamma^{2} \rho q_{n}$ & $\begin{array}{l}\text { prognostic variable of } n \text {-th component of mass } \\
\text { concentration }\end{array}$ & {$\left[\mathrm{kg} \mathrm{m}^{-3}\right]^{\dagger}$} \\
\hline$G_{A}$ & $\begin{array}{l}\text { tendency term of variable } A\left(A=R, \mathbf{V}_{h}, W \text {, }\right. \\
\left.E_{a}, Q_{n}, E_{a}+K+\Phi\right)\end{array}$ & {$\left[(\text { unit of } A) \times \mathrm{s}^{-1}\right]^{\dagger}$} \\
\hline$W_{n}^{*}=G^{1 / 2} \gamma^{2} \rho q_{n} w_{n}^{*}$ & precipitation mass flux of $n$-th component of wa- & {$\left[\mathrm{kg} \mathrm{m}^{-2} \mathrm{~s}^{-1}\right]^{\dagger}$} \\
\hline$\left(\tilde{\mathbf{F}}, \tilde{Q}_{\text {heat }}, \tilde{S}_{n}\right)=$ & $\begin{array}{l}\text { ter } \\
\text { source terms of momentum, energy, and } n \text {-the }\end{array}$ & \\
\hline$G^{1 / 2} \gamma^{2}\left(\mathbf{f}, q_{\text {heat }}, s_{n}\right)$ & component of mass concentration & \\
\hline$(\tilde{\mathbf{A}}, \tilde{\mathbf{C}})=G^{1 / 2} \gamma^{2}(\mathbf{a}, \mathbf{c})$ & advection of momentum and the Coriolis force & \\
\hline$(K, \Phi)=\rho G^{1 / 2} \gamma^{2}(k, \phi)$ & kinetic energy, potential energy & {$\left[\mathrm{kg} \mathrm{m}^{-1} \mathrm{~s}^{-2}\right]^{\dagger}$} \\
\hline
\end{tabular}

Table 1(5): Description of symbols in the text (cont.). $\dagger: G^{1 / 2}$ is assumed to be nondimensional. 


\begin{tabular}{|c|c|c|}
\hline symbol & description & unit \\
\hline$N_{g}$ & total grid number & \\
\hline$P_{i}(i=0, \cdots, 6)^{\S}$ & vertices of triangles & \\
\hline$Q_{i}(i=1, \cdots, 6)^{\S}$ & vertices of control volumes & \\
\hline$s_{0}, s_{1}, s_{2}$ & areas of triangles & {$\left[\mathrm{m}^{2}\right]$} \\
\hline$a\left(P_{0}\right)$ & area of control volume at $P_{0}$ & {$\left[\mathrm{~m}^{2}\right]$} \\
\hline$b_{i}(i=1, \cdots, 6)^{\S}$ & geodesic arc length of $Q_{i} Q_{i+1}{ }^{\ddagger}$ & {$[\mathrm{m}]$} \\
\hline $\mathbf{n}_{i}(i=1, \cdots, 6)^{\S}$ & outward unit vector at the midpoint of $Q_{i} Q_{i+1}{ }^{\ddagger}$ & \\
\hline$\left\{\mathbf{e}_{1}, \mathbf{e}_{2}, \mathbf{e}_{3}\right\}$ & orthogonal basis & \\
\hline$\left(v_{h 1}, v_{h 2}, v_{h 3}\right)=\mathbf{v}_{h} \cdot\left(\mathbf{e}_{1}, \mathbf{e}_{2}, \mathbf{e}_{3}\right)$ & components of horizontal velocity vector & {$\left[\mathrm{m} \mathrm{s}^{-1}\right]$} \\
\hline $\begin{array}{l}\left(V_{h 1}, V_{h 2}, V_{h 3}\right)= \\
\rho G^{1 / 2} \gamma^{2}\left(v_{h 1}, v_{h 2}, v_{h 3}\right)\end{array}$ & components of horizontal velocity vector & {$\left[\mathrm{kg} \mathrm{m}^{-2} \mathrm{~s}^{-1}\right]^{\dagger}$} \\
\hline$\Delta t$ & large time step interval & {$[\mathrm{s}]$} \\
\hline$\Delta \tau$ & small time step interval & {$[\mathrm{s}]$} \\
\hline$N_{s}=\Delta t / \Delta \tau$ & split number & \\
\hline$\phi^{*}=\phi-\phi^{t}$ & deviation of $\phi$ from its value at large time step $t$ & \\
\hline$\tilde{g}^{t}$ & modified gravity at $t$ & {$\left[\mathrm{~m} \mathrm{~s}^{-2}\right]$} \\
\hline$c_{s}=\left[\left(R_{d} / C_{v}\right) h_{a}\right]^{1 / 2}$ & acoustic wave speed & {$\left[\mathrm{m} \mathrm{s}^{-1}\right]$} \\
\hline
\end{tabular}

Table 1(6): Description of symbols in the text (cont.). $\dagger: G^{1 / 2}$ is assumed to be nondimensional. $\ddagger: i+1$ is replaced by 1 if $i=6 . \S: 6$ is replaced by 5 if the control volume is pentagon. 


\begin{tabular}{|c|c|c|}
\hline symbol & description & unit \\
\hline$D$ & three-dimensional divergence of momentum & {$\left[\mathrm{kg} \mathrm{m}^{-3} \mathrm{~s}^{-1}\right]$} \\
\hline$A_{d H}, A_{d V}$ & divergence damping coefficients & {$\left[\mathrm{m}^{4} \mathrm{~s}^{-1}\right]$} \\
\hline$\alpha_{d H}, \alpha_{d V}$ & non-dimensional divergence damping coefficients & \\
\hline$\overline{\Delta x}=\sqrt{4 \pi r_{0}^{2} / N_{g}}$ & mean horizontal grid interval & {$[\mathrm{m}]$} \\
\hline$K_{H}$ & lateral diffusion coefficient & {$\left[\mathrm{m}^{4} \mathrm{~s}^{-1}\right]$} \\
\hline$\gamma_{H}$ & non-dimensional lateral diffusion coefficient & \\
\hline$\tau_{d i v}=\overline{\Delta x}^{4} / A_{d H}$ & damping time of horizontal divergence damping & {$[\mathrm{s}]$} \\
\hline$\tau_{d i f}=\overline{\Delta x}^{4} / K_{H}$ & damping time of lateral diffusion & {$[\mathrm{s}]$} \\
\hline$\overline{\boldsymbol{V}}^{t}$ & average mass flux over large time step interval & {$\left[\mathrm{kg} \mathrm{m}^{-2} \mathrm{~s}^{-1}\right]$} \\
\hline
\end{tabular}
Table 1(7): Description of symbols in the text (cont.). 